\title{
Robust tracking control design for fractional-order interval type-2 fuzzy systems
}

\section{Kavikumar Ramasamy}

Chungbuk National University

Sakthivel Rathinasamy ( $\nabla$ krsakthivel@yahoo.com )

Bharathiar University https://orcid.org/0000-0002-5528-2709

Kwon O.M

Chungbuk National University

Selvaraj Palanisamy

Chungbuk National University

\section{Research Article}

Keywords: Fractional-order systems, Interval type-2 fuzzy model, Robust control design, Stability analysis.

Posted Date: July 19th, 2021

DOI: https://doi.org/10.21203/rs.3.rs-190103/v1

License: (9) This work is licensed under a Creative Commons Attribution 4.0 International License. Read Full License 


\title{
Robust tracking control design for fractional-order interval type-2 fuzzy systems
}

\author{
Ramasamy Kavikumar • Rathinasamy Sakthivel · Oh-Min Kwon • Palanisamy \\ Selvaraj
}

Received: date / Accepted: date

\begin{abstract}
This paper is concerned with an uncertainty and disturbance estimator-based tracking control problem for a class of interval type-2 fractional-order Takagi-Sugeno fuzzy systems subject to time-varying delays. The footprints of the uncertainty of the underlying fuzzy systems are taken into account to capture and model different levels of uncertainties. The uncertainty and disturbance estimator is used to promote the tracking behavior of rejecting disturbance in the control system. First, by applying the Lyapunov approach, we focus on the examination of stability and performance of the fractional-order tracking error system. Next, unknown system uncertainties, external disturbances and nonlinearities are accurately estimated via an appropriate filter design. Especially, the proposed control technique does not require any prior knowledge about above said unknown factors and it only requires the bandwidth information about the lowpass filter. Then, four numerical examples with simulation results are presented in the end, to show the potential of the theoretical results of the proposed control method.
\end{abstract}

Keywords Fractional-order systems - Interval type-2 fuzzy model $\cdot$ Robust control design $\cdot$ Stability analysis.

Ramasamy Kavikumar, Oh-Min Kwon $(\otimes) \cdot$ Palanisamy Selvaraj School of Electrical Engineering, Chungbuk National University, 1 Chungdae-ro, Cheongju 28644, South Korea.

Oh-Min Kwon

E-mail: madwind@chungbuk.ac.kr

Rathinasamy Sakthivel $(\otimes)$

Department of Applied Mathematics, Bharathiar University,

Coimbatore 641046, India

Department of Mathematics, Sungkyunkwan University,

Suwon 440-746, South Korea.

E-mail: krsakthivel@yahoo.com

\section{Introduction}

In recent few decades, the study of nonlinear control systems has paid much attention since many real-world happenings are governed by nonlinear differential and difference equations [1--4]. It is often very difficult to design accurate controllers for nonlinear systems directly due to their complicated dynamical behaviors. Recent studies of control theory have mainly resolved this issue with the aid of various advanced linearisation techniques, where the actual nonlinear systems are approximated as linear systems and then various linear control techniques are applied [5--7]. Among various linearisation approaches, Takagi-Sugeno (T-S) fuzzy model-based approach developed in [8] has been described as an important and successful method for linearizing of any smooth nonlinear system. Support of T-S fuzzy model technique, the nonlinear system is approximated as sum of a collection of linear subsystems with their weighting membership functions (MFs). Under this framework, a variety of linear type controllers are proposed for many nonlinear systems, which can be applied to various kinds of real-time applications [9-12].

The research of the T-S fuzzy model technique can represent the nonlinear information of the system effectively, but it shows limitations in dealing with the uncertainty that existing in the system. In order to handle this situation, the interval type-2 (IT2) fuzzy model-based approach are introduced. The main advantage of this approach is that it could efficiently handle the grades of membership uncertainties over fuzzy systems by capturing the effect of uncertainties through the upper and lower MFs. Recently, the IT2 fuzzy model strategies has been researched and some results have been produced [13-18]. In [14], the adaptive sliding mode controller has been proposed for IT2FSs to keep the closedloop system stable. Based on imperfect premise matching, the state space was split into two sub-spaces to enhance the 
stability analysis in the interval type-2 T-S fuzzy systems (IT2FSs) in [15].

Up to now, it was also well-known that one of the superiorities of fractional-order (FO) systems compared with integer-order systems are that FO systems can possess the capacity of infinite memory and also can express heredity and then can describe the real-world physical systems more accurately and precisely [19,20]. In particular, the general form of integer-order calculus as fractional calculus and it can be expected to provide a more accurate description of the system dynamics compared to classical integer-order models. Due to its potential applications of fractional calculus, the researches have been increased in many industrial and research fields, such as bioengineering, physics, economics and signal processing. Also, studies on FO system have recently gained significant attention from control research communities because of their potential applications in various engineering systems and control processes, such as stabilization, synchronization, state estimation and reference tracking [21-26]. Although many useful research works have been constructed based on fuzzy approach, most of them are concerned with integer-order systems and only a little amount of them are dealt with FO fuzzy systems [27, 28].

The modeling errors, parameter uncertainties, and external disturbances are unavoidable in real-time control systems, which may degrade systems efficiency and lead to instability. Therefore, uncertainties and external disturbances rejection is a key objective in the synthesis of modern control systems and several robust control techniques have been developed to handle the aforementioned factors in recent years [29-33]. At the early stage, high-gain feedback control approach is developed for many dynamical systems to compensate the disturbance effect [29], where the feedback control loop-gain needs to extend large value to get required control performance. In addition, many physical actuator devices fail to provide larger outputs, which will bring considerable practical difficulties in the process of high-gain control design. In order to avoid this difficulty, the integral control scheme is developed in [30], which could attenuate the constant external disturbance effect without increasing gain value largely but it may not be suitable when the time-varying disturbance exists. Recently, the sliding mode control approach is constructed for motion control system in [33], which effectively handles uncertainty, constants and time-varying disturbances but it requires the bound value of the external disturbance and its derivative. In some practical situations, it may be difficult to get enough the information about external disturbances and uncertainties. To handle this issue, many modern control techniques are developed to estimate them [34-37]. Among such techniques, uncertainty and disturbance estimator (UDE)-based control design proposed by Zhong and Rees in [36], has been widely used in many industrial and engineering applications [37]. This approach not only compensates the uncertainty and disturbance effect but also helps linearise highly nonlinear systems. In addition, it does not require any information about the disturbance and system uncertainties, which is practically more important and significant.

Motivated by the aforementioned studies and achievements, in this paper, a robust tracking problem is considered for FO IT2FSs via a UDE-based control strategy. Moreover, the main contributions and advantages of the proposed study are summarized as follows:

(i) The robust tracking problem of UDE-based control strategy is first time proposed for FO IT2FSs with unknown uncertainties, nonlinearities, and external disturbances.

(ii) The proposed UDE-based control design relaxes the general assumption considered on unknown uncertainties, nonlinearities and external disturbances since it requires only its bandwidth information. Also, proposed method has significantly fewer tuning parameters, so it is easy to implement in many practical systems such as aerial vehicles, underwater vehicles, and satellites.

(iii) To facilitate the stability analysis, the lower and upper MFs of plant and controller are considered within the footprint of uncertainty (FOU), which leads to obtain less conservative stability result.

(iv) The stability criterion of the designed method is derived by employing a Lyapunov- Krasovskii functional method, slack matrix and linear matrix inequalities. In order to demonstrate the validity of the developed theoretical results, three numerical examples are presented.

The paper is organized as follows: FO IT2FSs model, FO IT2 fuzzy reference and FO IT2 fuzzy error dynamics are introduced systematically in Section 2 Section 3 provides performance analysis results for FO IT2 fuzzy error dynamics. The corresponding simulation results are provided in Section 4 . Conclusions and future works are given in Section 5

Notation: Throughout this paper, $\mathbb{Z}^{+}$is the set of all positive integers. $\mathbb{R}^{n}$ and $\mathbb{R}^{n \times m}$ are, respectively, the $n$ dimensional vector space and the $n \times m$ real matrix space. ' $*$ ' represents the symmetric terms in a symmetric matrix.

' $\star$ ' indicates the convolution operator. $\mathcal{B}_{l}^{+}$is the pseudoinverse of $\mathcal{B}_{l} \cdot \mathcal{L}^{-1}\{\cdot\}$ stands for the inverse Laplace transform operator. The superscripts " $T$ " and " -1 " indicate matrix transposition and matrix inverse, respectively.

\section{Problem formulation}

As presented in [38], Caputo derivative is well understood in physical situations and more applicable to real-world problems. Therefore, in this paper, we consider the following $\mathrm{Ca}$ puto fractional derivative. 
Definition 1 [38] The Caputo fractional derivative of FO $\alpha$ of function $f(t)$ is defined as

$\mathcal{D}^{\alpha} f(t)=\frac{1}{\Gamma(i-\alpha)} \frac{d^{i}}{d t^{i}} \int_{t_{0}}^{t}(t-\Phi)^{i-\alpha-1} f(\Phi) d \Phi$,

where $\alpha$ represents the order of the derivative, such that $0 \leq$ $i-1 \leq \alpha<i, i \in \mathbb{Z}^{+}$and $\Gamma(j)=\int_{0}^{\infty} t^{j-1} e^{-1} d t$.

Remark 1 It should be mentioned that the Laplace transform of the Caputo fractional derivative requires for the use of initial values of integer-order derivatives with clear physical representations in practical theoretical models, whereas the Riemann-Liouville fractional derivative does not. In general, the Caputo fractional differential equation is appropriate for analyzing real dynamic models with non-zero initial values, whereas the Riemann-Liouville fractional differential equation is better suited for presenting fractional-order systems with a static initial state. As a result, the Caputo operator is utilized in this work to investigate fractional-order IT2FSs.

Consider the following FO IT2FSs with $\bar{m}$ fuzzy rules subject to unknown uncertainties, nonlinearities and external disturbances:

Plant rule $l: \operatorname{IF} \bar{\delta}_{1}(\rho(t))$ is $\overline{\mathbb{E}}_{1}^{l}, \bar{\delta}_{2}(\rho(t))$ is $\overline{\mathbb{E}}_{2}^{l}, \ldots, \bar{\delta}_{\psi}(\rho(t))$ is $\overline{\mathbb{E}}_{\psi}^{l}$ THEN

$$
\begin{aligned}
\mathcal{D}^{\alpha} \rho(t)= & \left(\mathcal{A}_{l}+\Delta \mathcal{A}_{l}(t)\right) \rho(t)+\mathcal{A}_{l \tau} \rho(t-\tau(t))+\mathcal{B}_{l} \vartheta(t) \\
& +\mathcal{C}_{l} \beta(t)+g(\rho(t))
\end{aligned}
$$

where $\overline{\mathbb{E}}_{\tilde{\Omega}}^{l}(l \in \mathbb{L}=\{1,2, \ldots, \bar{m}\}, \tilde{\Omega}=1,2, \ldots, \psi)$ denotes the IT2 fuzzy set with the integer $\psi>0 ; \bar{\delta}_{\tilde{\Omega}}(\rho(t))$ denotes the measurable premise variable; $\rho(t) \in \mathbb{R}^{n}$ indicates the system state vector; $\vartheta(t) \in \mathbb{R}^{n_{\vartheta}}$ is the control input vector; $g(\rho(t)) \in \mathbb{R}^{n_{g}}$ denotes the unknown nonlinear function; $\tau(t)$ is time-varying delay and satisfy the conditions $0 \leq \tau(t) \leq \tau$, where $\tau$ are known positive constants; $\beta(t) \in \mathbb{R}^{n_{\beta}}$ stands for external disturbance input. Further, $\mathcal{A}_{l}, \mathcal{A}_{l \tau}, \mathcal{B}_{l}$ and $\mathcal{C}_{l}$ represent known system matrices; $\Delta \mathcal{A}_{l}(t)$ is the unknown time-varying uncertainty matrix. The following interval set presents the firing strength of the $l$-th fuzzy rule:

$$
\mathbb{M}_{l}(\rho(t))=\left[\underline{\lambda}_{l}(\rho(t)), \bar{\lambda}_{l}(\rho(t))\right], l \in \mathbb{L},
$$

where the nonlinear functions $\underline{\lambda}_{l}(\rho(t))$ and $\bar{\lambda}_{l}(\rho(t))$ stand for lower and upper grade of MFs of the $l$-th fuzzy rule, respectively. Further, they satisfy $0 \leq \underline{\lambda}_{l}(\rho(t))=$ $\prod_{\tilde{\Omega}=1}^{\psi}{\underline{\overline{\mathbb{E}}_{\tilde{\Omega}}^{l}}}_{\tilde{\Omega}}\left(\bar{\delta}_{\tilde{\Omega}}(\rho(t))\right) \leq \bar{\lambda}_{l}(\rho(t))=\prod_{\tilde{\Omega}=1}^{\psi} \bar{\zeta}_{\overline{\mathbb{E}}_{\tilde{\Omega}}^{l}}\left(\bar{\delta}_{\tilde{\Omega}}(\rho(t))\right)$ in which the positive functions ${\underline{\zeta_{\bar{E}^{l}}}}_{\tilde{\delta}}\left(\bar{\delta}_{\tilde{\Omega}}(\rho(t))\right), \bar{\zeta}_{\overline{\mathbb{E}}_{\tilde{\Omega}}^{l}}\left(\bar{\delta}_{\tilde{\Omega}}(\rho(t))\right)$ denote the lower and upper MFs of $l$-th fuzzy rule, respectively.
Then, the overall FO fuzzy system is represented by

$$
\begin{aligned}
\mathcal{D}^{\alpha} \rho(t) & =\sum_{l=1}^{\bar{m}} \lambda_{l}(\rho(t))\left[\left(\mathcal{A}_{l}+\Delta \mathcal{A}_{l}(t)\right) \rho(t)+\mathcal{B}_{l} \vartheta(t)\right. \\
& \left.+\mathcal{A}_{l \tau} \rho(t-\tau(t))+\mathcal{C}_{l} \beta(t)\right]+g(\rho(t)),
\end{aligned}
$$

where $\lambda_{l}(\rho(t))=y_{l}(\rho(t)) \underline{\lambda}_{l}(\rho(t))+\bar{y}_{l}(\rho(t)) \bar{\lambda}_{l}(\rho(t))$ with $\bar{y}_{l}(\rho(t))$ and $y_{l}(\rho(t))$ being nonlinear weighting functions and they satisfy $0 \leq \bar{y}_{l}(\rho(t)), \underline{y}_{l}(\rho(t)) \leq 1$ and $\underline{y}_{l}(\rho(t))+$ $\bar{y}_{l}(\rho(t))=1$. Further, $\lambda_{l}(\rho(t)), \forall l \in \mathbb{L}$ indicates the grades of MFs with $\sum_{l=1}^{\bar{m}} \lambda_{l}(\rho(t))=1$.

Next, let us consider the following FO fuzzy reference model:

$$
\begin{aligned}
\mathcal{D}^{\alpha} \rho_{m}(t) & =\sum_{a=1}^{\bar{m}} \lambda_{a}\left(\rho_{m}(t)\right)\left[\mathcal{A}_{a m} \rho_{m}(t)+\mathcal{B}_{a m} r(t)\right. \\
& \left.+\mathcal{A}_{a m \tau} \rho_{m}(t-\tau(t))\right], \quad a \in \mathbb{L},
\end{aligned}
$$

where $\rho_{m}(t) \in \mathbb{R}^{n}$ indicates the reference state vector; $r(t)=$ $\left[r_{1}(t), r_{2}(t), \ldots, r_{p}(t)\right]^{T} \in \mathbb{R}^{p}$ is the piecewise continuous and uniformly bound command to the reference model; $\mathcal{A}_{a m}, \mathcal{A}_{a m \tau}$ and $\mathcal{B}_{a m}$ are known constant matrices.

The objective of this work is to determine a state feedback control $\vartheta(t)$ for the system (2) that forces the error vector $e(t)=\rho_{m}(t)-\rho(t)$ between the system and the reference model to zero. That is, the desired error dynamics in the form

$$
\begin{aligned}
\mathcal{D}^{\alpha} e(t) & =\sum_{a=1}^{\bar{m}} \sum_{b=1}^{\bar{n}} \lambda_{a}\left(\rho_{m}(t)\right) \mu_{b}\left(\rho_{m}(t)\right)\left[\left(\mathcal{A}_{a m}+\mathcal{K}_{b}\right) e(t)\right. \\
& \left.+\mathcal{A}_{a m \tau} e(t-\tau(t))\right]
\end{aligned}
$$

should be asymptotically stable, where $\mathcal{K}_{b}(b \in \mathbb{H}$ $=\{1,2, \ldots, \bar{n}\})$ are the feedback control gain matrices and $\mu_{b}\left(\rho_{m}(t)\right)=\frac{\underline{z}_{b}\left(\rho_{m}(t)\right) \underline{\mu}_{b}\left(\rho_{m}(t)\right)+\bar{z}_{b}\left(\rho_{m}(t)\right) \bar{\mu}_{b}\left(\rho_{m}(t)\right)}{\sum_{b=1}^{\bar{n}}\left(\underline{z}_{b}\left(\rho_{m}(t)\right) \underline{\mu}_{b}\left(\rho_{m}(t)\right)+\bar{z}_{b}\left(\rho_{m}(t)\right) \bar{\mu}_{b}\left(\rho_{m}(t)\right)\right)}$ in which $\underline{z}_{b}\left(\rho_{m}(t)\right)$ and $\bar{z}_{b}\left(\rho_{m}(t)\right)$ denote the lower and upper nonlinear weighting functions, respectively.

From the equations (2)-(4), we have the following control relation:

$$
\begin{aligned}
\vartheta(t)= & \sum_{l=1}^{\bar{m}} \sum_{a=1}^{\bar{m}} \sum_{b=1}^{\bar{n}} \lambda_{l}(\rho(t)) \lambda_{a}\left(\rho_{m}(t)\right) \mu_{b}\left(\rho_{m}(t)\right) \mathcal{B}_{l}^{+} \\
& \times\left[-\mathcal{K}_{b} e(t)+\mathcal{A}_{a m} \rho(t)+\mathcal{B}_{a m} r(t)-\mathcal{A}_{l} \rho(t)\right. \\
& \left.+\left(\mathcal{A}_{a m \tau}-\mathcal{A}_{l \tau}\right) \rho(t-\tau(t))-\hat{\beta}(t, \rho(t))\right],
\end{aligned}
$$

where $\mathcal{B}_{l}^{+}=\left(\mathcal{B}_{l}^{T} \mathcal{B}_{l}\right)^{-1} \mathcal{B}_{l}^{T}$ is the pseudo-inverse of $\mathcal{B}_{l}$ and $\hat{\beta}(t, \rho(t))=\sum_{l=1}^{\bar{m}} \lambda_{l}(\rho(t))\left(\Delta A_{l}(t)+\mathcal{C}_{l} \beta(t)\right)+g(\rho(t))$ is the 
lumped unknown nonlinearity, which is the sum of uncertainty, unknown nonlinear dynamics and unknown external disturbance.

It is noted that the control law (5) cannot be used directly because control expression contains unknown lumped dynamic elements. Hence, it is necessary to estimate the state with the aid of known factors to access the control law (5). According to the considered FO IT2FSs dynamics (2), the lumped unknown nonlinearity term $\hat{\beta}(t, \rho(t))$ can be represented as

$$
\begin{aligned}
\hat{\beta}(t, \rho(t))= & \mathcal{D}^{\alpha} \rho(t)-\sum_{l=1}^{\bar{m}} \lambda_{l}(\rho(t))\left\{\mathcal{A}_{l} \rho(t)+\mathcal{B}_{l} \vartheta(t)\right. \\
& \left.+\mathcal{A}_{l \tau} \rho(t-\tau(t))\right\},
\end{aligned}
$$

which demonstrates that the unknown lumped nonlinearity can be obtained from the known dynamics of the system and control signal. But it cannot be directly accessible to formulate a control law since it depends on the fractional derivative of the state vector. According to UDE-based control scheme [37], an estimation of this signal is adopted based on the assumption that a signal can be approximated and estimated using a filter with the appropriate bandwidth.

To accurately approximate the $\hat{\beta}(t, \rho(t))$, the fractional low-pass filter $\mathcal{H}_{f}(s)=\frac{1}{\mathcal{T}_{k} s^{\alpha}+1}$ is adopted, where the small positive scalar $\mathcal{T}_{k}=\frac{1}{\omega_{c}}$, in which $\omega_{c}$ represents the cut-off frequency of the filter $\mathcal{H}_{f}(s)$. Now, the unknown lumped nonlinearity passes into the filter $\mathcal{H}_{f}(s)$ and the accurately estimated filtered output is expressed as follows:

$$
\begin{array}{r}
\bar{\beta}(t, \rho(t))=\hat{\beta}(t, \rho(t)) \star h_{f}(t)=\left[\mathcal{D}^{\alpha} \rho(t)-\sum_{l=1}^{\bar{m}} \lambda_{l}(\rho(t))\right. \\
\left.\times\left(\mathcal{A}_{l} \rho(t)+\mathcal{A}_{l \tau} \rho(t-\tau(t))+\mathcal{B}_{l} \vartheta(t)\right)\right] \star h_{f}(t),
\end{array}
$$

where $h_{f}(t)$ indicates the impulse response of $\mathcal{H}_{f}(s)$.

Further, replacing the unknown lumped nonlinearity $\hat{\beta}(t, \rho(t))$ in (5) by estimated lumped nonlinearity in (7) and by simple calculation, the control law is expressed as

$$
\begin{aligned}
\vartheta(t)= & \sum_{l=1}^{\bar{m}} \sum_{a=1}^{\bar{m}} \sum_{b=1}^{\bar{n}} \lambda_{l}(\rho(t)) \lambda_{a}\left(\rho_{m}(t)\right) \mu_{b}\left(\rho_{m}(t)\right) \mathcal{B}_{l}^{+} \\
& \times\left[\mathcal { L } ^ { - 1 } \{ \frac { 1 } { 1 - \mathcal { H } _ { f } ( s ) } \} \star \left(-\mathcal{K}_{b} e(t)+\mathcal{A}_{a m} \rho(t)\right.\right. \\
& \left.+\mathcal{B}_{a m} r(t)+\mathcal{A}_{a m \tau} \rho(t-\tau(t))\right)-\mathcal{A}_{l \tau} \rho(t-\tau(t)) \\
& \left.-\mathcal{A}_{l} \rho(t)-\mathcal{L}^{-1}\left\{\frac{s^{\alpha} \mathcal{H}_{f}(s)}{1-\mathcal{H}_{f}(s)}\right\} \star \rho(t)\right],
\end{aligned}
$$

where $\mathcal{L}^{-1}\{\cdot\}$ is the inverse Laplace transform operator. Then, we can obtain the resulting control law as follows:

$\vartheta(t)=\sum_{l=1}^{\bar{m}} \sum_{a=1}^{\bar{m}} \sum_{b=1}^{\bar{n}} \lambda_{l}(\rho(t)) \lambda_{a}\left(\rho_{m}(t)\right) \mu_{b}\left(\rho_{m}(t)\right) \mathcal{B}_{l}^{+}$

$$
\begin{aligned}
& \times\left[( 1 + \frac { 1 } { \mathcal { T } _ { k } } \mathcal { I } ^ { \alpha } ) \left(\mathcal{A}_{a m} \rho(t)+\mathcal{B}_{a m} r(t)-\mathcal{K}_{b} e(t)\right.\right. \\
& \left.+\mathcal{A}_{a m \tau} \rho(t-\tau(t))\right)-\mathcal{A}_{l} \rho(t)-\mathcal{A}_{l \tau} \rho(t-\tau(t)) \\
& \left.-\frac{1}{\mathcal{T}_{k}} \rho(t)\right]
\end{aligned}
$$

where $\mathcal{I}^{\alpha}$ is the Caputo fractional integral operator with or$\operatorname{der} \alpha$.

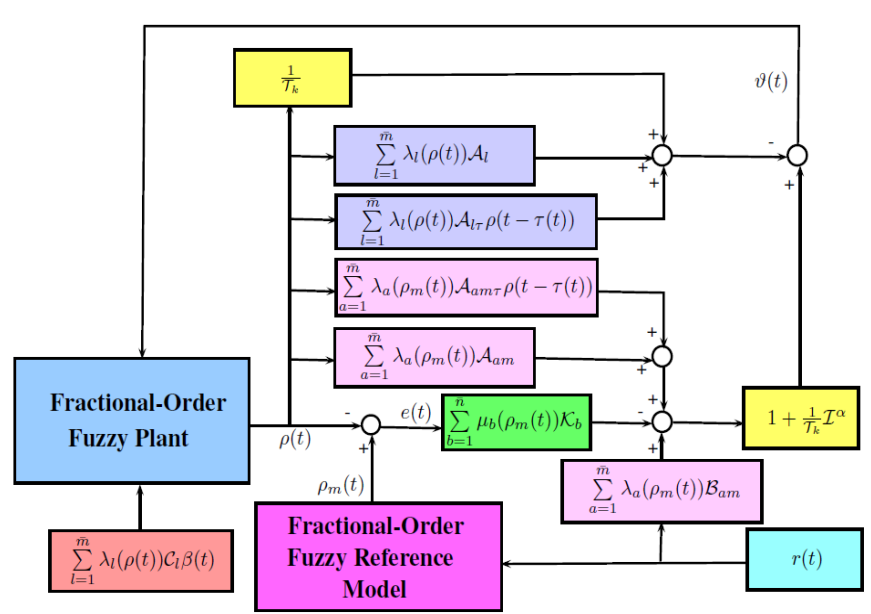

Fig. 1 Configuration of fractional-order fuzzy UDE-based control systems

Fig. 1 presents the frame works of the fuzzy system (2) and reference model (3) along with UDE-based control model (9).

In order to complete the system description, the following assumptions are more essential.

Assumption 1 The bandwidth of unknown lumped disturbance signal is known.

Assumption 2 The control coefficient matrices of each fuzzy rule of the system (2) have full column rank.

Remark 2 From Assumption 1 the bandwidth of the lumped disturbance signal is only required to estimate that unknown term. This assumption is easily guaranteed for most of the practical systems. Besides that, Assumption 2 is required for the existence of the pseudo inverse $B_{l}^{+}$. It should be noted that if the pseudo inverse exists then each control action has a various effect on the system. Otherwise, the choice of the reference model and the error feedback gain matrix is restricted.

Moreover, we consider the IT2FSs and controllers of the upper and lower MFs are recommended within the FOU. The state $\Theta$ is split into $\gamma$ connected sub-state spaces designed by $\Theta_{v}$ in which $\gamma$ is the number of sub-states which 
implies $\Theta=\cup_{v=1}^{\gamma} \Theta_{v}$. The FOU is partitioned into $\varrho+1$ sub-FOUs, for $c \in \mathbb{J}=\{1,2, \ldots, \varrho+1\}$. Then, the upper and lower MFs are defined as follows:

$$
\begin{aligned}
\bar{\chi}_{a b c}\left(\rho_{m}(t)\right)= & \sum_{v=1}^{\gamma} \sum_{a_{1}=1}^{2} \sum_{a_{2}=1}^{2} \cdots \sum_{a_{n}=1}^{2} \prod_{j=1}^{p} w_{j a_{j} v c}\left(\rho_{m j}(t)\right) \\
& \times \bar{\varepsilon}_{a b a_{1} a_{2} \ldots a_{n} v c} \\
\underline{\chi}_{a b c}\left(\rho_{m}(t)\right)= & \sum_{v=1}^{\gamma} \sum_{a_{1}=1}^{2} \sum_{a_{2}=1}^{2} \cdots \sum_{a_{n}=1}^{2} \prod_{j=1}^{p} w_{j a_{j} v l}\left(\rho_{m j}(t)\right) \\
& \times \underline{\varepsilon}_{a b a_{1} a_{2} \ldots a_{n} v c} \\
\sum_{v=1}^{\gamma} \sum_{a_{1}=1}^{2} \sum_{a_{2}=1}^{2} \cdots & \sum_{a_{n}=1}^{2} \prod_{j=1}^{p} w_{j a_{j} v c}\left(\rho_{j}(t)\right)=1
\end{aligned}
$$

where $0 \leq w_{j a_{m j} v c}\left(\rho_{m j}(t)\right) \leq 1, w_{j 1 v c}\left(\rho_{m j}(t)\right)$ $+w_{j 2 v c}\left(\rho_{m j}(t)\right)=1, \forall j=1,2, \ldots, p, 0 \leq \underline{\chi}_{a b c}\left(\rho_{m}(t)\right) \leq$ $\bar{\chi}_{a b c}\left(\rho_{m}(t)\right) \leq 1, \bar{\varepsilon}_{a b a_{1} a_{2} \ldots a_{n} v c}$ and $\underline{\varepsilon}_{a b a_{1} a_{2} \ldots a_{n} v c}$ are scalars to be determined and $0 \leq \underline{\varepsilon}_{a b a_{1} a_{2} \ldots a_{n} v c} \leq \bar{\varepsilon}_{a b a_{1} a_{2} \ldots a_{n} v c} \leq$ 1 , for $j=1,2, \ldots, p, a_{j}=1,2, v=1,2, \ldots, \gamma$ and $\rho_{m}(t) \in \Theta_{v}$.

Remark 3 It should be noted that the proposed controller (9) has only two degrees of freedom, namely error feedback gain matrices $\mathcal{K}_{b}, b=1,2, \ldots, \bar{n}$, and the filter parameter $\mathcal{T}_{k}$ and their selection is independent of each other. More precisely, the feedback control gain matrices can be easily obtained from the solution of the stability problem of nominal error system (4). Moreover, $\mathcal{T}_{k}$, s value depends on the specified application including the hardware capability and the performance requirements, which can be easily identified based on the assumption that bandwidth information of the unknown lumped nonlinear signal is known. In particular, if the filter has a wide enough bandwidth, the UDE is able to accurately and quickly estimate the lumped uncertainty.

\section{Main results}

In this section, the issue of robust tracking control design for error system (4) is considered. To be precise, based on the UDE-based controller (9), a tracking control is designed to guarantee the asymptotic stability of the states of error systems (4). In particular, some slack matrices under the $S$ - procedure [18] are employed in the derivation of obtaining required stability conditions. For representation convenience, we denote: $\nu_{a b c}\left(\rho_{m}(t)\right)=\nu_{a b c}, \underline{\kappa}_{a b c}\left(\rho_{m}(t)\right)=$ $\underline{\kappa}_{a b c}, \bar{\kappa}_{a b c}\left(\rho_{m}(t)\right)=\bar{\kappa}_{a b c}, \underline{\chi}_{a b c}\left(\rho_{m}(t)\right)=\underline{\chi}_{a b c}$, $\bar{\chi}_{a b c}\left(\rho_{m}(t)\right)=\bar{\chi}_{a b c}, \sum_{a=1}^{\bar{m}} \sum_{b=1}^{\bar{n}} \lambda_{a}\left(\rho_{m}(t)\right) \mu_{b}\left(\rho_{m}(t)\right)=$ $\sum_{a=1}^{\bar{m}} \sum_{b=1}^{\bar{n}} \sum_{c=1}^{\varrho+1} \nu_{a b c}\left(\underline{\kappa}_{a b c} \underline{\chi}_{a b c}+\bar{\kappa}_{a b c} \bar{\chi}_{a b c}\right)$.
Theorem 1 For the given scalars $\tau, \alpha \in(0,1]$, the FO IT2 fuzzy error system (4) is asymptotically stable, if there exist symmetric positive-definite matrices $\overline{\mathcal{P}} \in \mathbb{R}^{n \times n}, \mathcal{V}_{a b c} \in$ $\mathbb{R}^{n \times n}$, symmetric matrix $\mathcal{U} \in \mathbb{R}^{n \times n},(a \in \mathbb{L}, b \in \mathbb{H}, c \in \mathbb{J})$ and matrices $\mathcal{X}_{b}(b \in \mathbb{H})$ such that

$$
\begin{aligned}
& \Upsilon_{a b}+\mathcal{V}_{a b c}+\mathcal{U}>0, \forall a, b, c \\
& \sum_{a=1}^{\bar{m}} \sum_{b=1}^{\bar{n}} \sum_{c=1}^{\varrho+1}\left[\bar{\varepsilon}_{a b a_{1} a_{2} \ldots a_{n} v c} \Upsilon_{a b}-\left(\underline{\varepsilon}_{a b a_{1} a_{2} \ldots a_{n} v c}\right.\right. \\
& \left.\left.-\bar{\varepsilon}_{a b a_{1} a_{2} \ldots a_{n} v c}\right) \mathcal{V}_{a b c}+\bar{\varepsilon}_{a b a_{1} a_{2} \ldots a_{n} v c} \mathcal{U}\right]-\mathcal{U}<0, \forall v, c
\end{aligned}
$$

where

$\Upsilon_{a b}=\left[\begin{array}{ccc}\Upsilon_{a b}^{1} & \Upsilon_{a b}^{2} & \Upsilon_{a b}^{4} \\ * & \Upsilon_{a b}^{3} & \Upsilon_{a b}^{5} \\ * & * & \Upsilon_{a b}^{6}\end{array}\right], \mathcal{V}_{a b c}=\left[\begin{array}{ccc}\mathcal{V}_{1 a b c} & \mathcal{V}_{2 a b c} & \mathcal{V}_{3 a b c} \\ * & \mathcal{V}_{4 a b c} & \mathcal{V}_{5 a b c} \\ * & * & \mathcal{V}_{6 a b c}\end{array}\right]$

$\mathcal{U}=\left[\begin{array}{ccc}\mathcal{U}_{1} & \mathcal{U}_{2} & \mathcal{U}_{3} \\ * & \mathcal{U}_{4} & \mathcal{U}_{5} \\ * & * & \mathcal{U}_{6}\end{array}\right], \Upsilon_{a b}^{1}=\operatorname{Sym}\left(\mathcal{A}_{a m} \overline{\mathcal{P}}+\mathcal{X}_{b}\right)+\tau^{\alpha} \alpha^{-1} \overline{\mathcal{P}}+$ $\overline{\mathcal{P}}, \Upsilon_{a b}^{2}=\mathcal{A}_{a m \tau} \overline{\mathcal{P}}, \Upsilon_{a b}^{3}=-\overline{\mathcal{P}}, \Upsilon_{a b}^{4}=\tau^{\alpha} \alpha^{-1} \mathcal{A}_{a m}^{T} \overline{\mathcal{P}}+$ $\tau^{\alpha} \alpha^{-1} \mathcal{X}_{b}, \Upsilon_{a b}^{5}=\tau^{\alpha} \alpha^{-1} \mathcal{A}_{a m \tau}$, and $\Upsilon_{a b}^{6}=-\tau^{\alpha} \alpha^{-1}$ I. Moreover, the control gain matrices can be obtained by $\mathcal{K}_{b}=$ $\overline{\mathcal{P}}-1 \mathcal{X}_{b}$.

Proof: We choose a Lyapunov-Krasovskii function for error system (4) as

$\mathcal{W}(e(t))=e^{T}(t) \mathcal{P} e(t)$.

The derivative of the Lyapunov-Krasovskii functional can be computed in the view of [38].

$$
\begin{aligned}
D^{\alpha} \mathcal{W}(e(t)) \leq & \sum_{a=1}^{\bar{m}} \sum_{b=1}^{\bar{n}} \lambda_{a}\left(\rho_{m}(t)\right) \mu_{b}\left(\rho_{m}(t)\right) \\
& \times\left\{e ^ { T } ( t ) \left(\mathcal{P}\left(\mathcal{A}_{a m}+\mathcal{K}_{b}\right)\right.\right. \\
& \left.+\left(\mathcal{A}_{a m}+\mathcal{K}_{b}\right)^{T} \mathcal{P}\right) e(t) \\
& \left.+e^{T}(t) \mathcal{P} \mathcal{A}_{a m \tau} e(t-\tau(t))\right\}
\end{aligned}
$$

On the other hand, the following inequality holds:

$$
\begin{aligned}
& \tau^{\alpha} \alpha^{-1}\left[\begin{array}{c}
e(t) \\
D^{\alpha}(e(t))
\end{array}\right]^{T}\left[\begin{array}{ll}
\mathcal{P} & 0 \\
0 & I
\end{array}\right]\left[\begin{array}{c}
e(t) \\
D^{\alpha}(e(t))
\end{array}\right] \\
& -\int_{t-\tau(t)}^{t}(t-s)^{\alpha-1}\left[\begin{array}{c}
e(t) \\
D^{\alpha}(e(t))
\end{array}\right]^{T}\left[\begin{array}{ll}
\mathcal{P} & 0 \\
0 & I
\end{array}\right]\left[\begin{array}{c}
e(t) \\
D^{\alpha}(e(t))
\end{array}\right] d s \geq 0 .
\end{aligned}
$$

Form 16 and 17, we have

$$
\begin{aligned}
D^{\alpha} \mathcal{W}(e(t)) \leq & \sum_{a=1}^{\bar{m}} \sum_{b=1}^{\bar{n}} \lambda_{a}\left(\rho_{m}(t)\right) \mu_{b}\left(\rho_{m}(t)\right) \\
& \times\left\{e ^ { T } ( t ) \left(\mathcal{P}\left(\mathcal{A}_{a m}+\mathcal{K}_{b}\right)\right.\right.
\end{aligned}
$$




$$
\begin{aligned}
& \left.+\left(\mathcal{A}_{a m}+\mathcal{K}_{b}\right)^{T} \mathcal{P}\right) e(t) \\
& \left.+e^{T}(t) \mathcal{P} \mathcal{A}_{a m \tau} e(t-\tau(t))\right\} \\
& +\tau^{\alpha} \alpha^{-1} e^{T}(t) \mathcal{P} e(t) \\
& +\tau^{\alpha} \alpha^{-1} D^{\alpha}(e(t))^{T} I D^{\alpha}(e(t)) \\
& -\int_{t-\tau(t)}^{t}(t-s)^{\alpha-1}\left[\begin{array}{c}
e(t) \\
D^{\alpha}(e(t))
\end{array}\right]^{T} \\
& \times\left[\begin{array}{cc}
\mathcal{P} & 0 \\
0 & I
\end{array}\right]\left[\begin{array}{c}
e(t) \\
D^{\alpha}(e(t))
\end{array}\right] d s .
\end{aligned}
$$

Since $e(t)$ satisfies for $-\tau \leq \theta \leq 0$,

$\mathcal{W}(t+\theta, e(t+\theta)) \leq p \mathcal{W}(t, e(t))$,

for some $p>1$, one can conclude that

$p e^{T}(t) \mathcal{P} e(t)-e^{T}(t-\tau(t)) \mathcal{P} e(t-\tau(t)) \geq 0$.

Combining (18) and 20, one has

$$
\begin{aligned}
& D^{\alpha} \mathcal{W}(e(t)) \leq \sum_{a=1}^{\bar{m}} \sum_{b=1}^{\bar{n}} \lambda_{a}\left(\rho_{m}(t)\right) \mu_{b}\left(\rho_{m}(t)\right) \\
& \times\left\{e ^ { T } ( t ) \left(\mathcal{P}\left(\mathcal{A}_{a m}+\mathcal{K}_{b}\right)\right.\right. \\
& \left.+\left(\mathcal{A}_{a m}+\mathcal{K}_{b}\right)^{T} \mathcal{P}+\tau^{\alpha} \alpha^{-1} \mathcal{P}\right) e(t) \\
& +e^{T}(t) \mathcal{P} \mathcal{A}_{a m \tau} e(t-\tau(t)) \\
& +\tau^{\alpha} \alpha^{-1}\left(\left(\left(\mathcal{A}_{a m}+\mathcal{K}_{b}\right) e(t)\right.\right. \\
& \left.+\mathcal{A}_{a m \tau} e(t-\tau(t))\right)^{T} I\left(\left(\mathcal{A}_{a m}+\mathcal{K}_{b}\right) e(t)\right. \\
& \left.\left.+\mathcal{A}_{a m \tau} e(t-\tau(t))\right)\right) \\
& -\int_{t-\tau(t)}^{t}(t-s)^{\alpha-1}\left[\begin{array}{c}
e(t) \\
D^{\alpha}(e(t))
\end{array}\right]^{T}\left[\begin{array}{ll}
\mathcal{P} & 0 \\
0 & I
\end{array}\right] \\
& \times\left[\begin{array}{c}
e(t) \\
D^{\alpha}(e(t))
\end{array}\right] d s+p e^{T}(t) \mathcal{P} e(t) \\
& \left.-e^{T}(t-\tau(t)) \mathcal{P} e(t-\tau(t))\right\} \\
& =-\int_{t-\tau(t)}^{t}(t-s)^{\alpha-1}\left[\begin{array}{c}
e(t) \\
D^{\alpha}(e(t))
\end{array}\right]^{T}\left[\begin{array}{ll}
\mathcal{P} & 0 \\
0 & I
\end{array}\right] \\
& \times\left[\begin{array}{c}
e(t) \\
D^{\alpha}(e(t))
\end{array}\right] d s+\sum_{a=1}^{\bar{m}} \sum_{b=1}^{\bar{n}} \sum_{c=1}^{\varrho+1} \nu_{a b c} \\
& \times\left(\underline{\kappa}_{a b c} \underline{\chi}_{a b c}+\bar{\kappa}_{a b c} \bar{\chi}_{a b c}\right) \Sigma^{T}(t) \tilde{\Upsilon}_{a b} \Sigma(t),
\end{aligned}
$$

where

$\Sigma^{T}(t)=\left[\begin{array}{c}e^{T}(t) \\ e^{T}(t-\tau(t))\end{array}\right]^{T}, \tilde{\Upsilon}_{a b}=\left[\begin{array}{cc}\tilde{\Upsilon}_{a b}^{1} & \tilde{\Upsilon}_{a b}^{2} \\ * & \tilde{\Upsilon}_{a b}^{3}\end{array}\right], \tilde{\Upsilon}_{a b}^{1}=\mathcal{P}\left(\mathcal{A}_{a m}+\right.$ $\left.\mathcal{K}_{b}\right)+\left(\mathcal{A}_{a m}+\mathcal{K}_{b}\right)^{T} \mathcal{P}+\tau^{\alpha} \alpha^{-1} \mathcal{P}+p \mathcal{P}+\tau^{\alpha} \alpha^{-1}\left(\mathcal{A}_{a m}+\right.$ $\left.\mathcal{K}_{b}\right)^{T} I\left(\mathcal{A}_{a m}+\mathcal{K}_{b}\right), \tilde{\Upsilon}_{a b}^{2}=\mathcal{P} \mathcal{A}_{a m \tau}+\tau^{\alpha} \alpha^{-1}\left(\mathcal{A}_{a m}+\mathcal{K}_{b}\right) \mathcal{A}_{a m \tau}$ and $\tilde{\Upsilon}_{a b}^{3}=-\mathcal{P}+\tau^{\alpha} \alpha^{-1} \mathcal{A}_{a m \tau}^{T} \mathcal{A}_{a m \tau}$.
By applying the Schur complement to $\tilde{\Upsilon}_{a b}$ is equivalent written as

$\bar{\Upsilon}_{a b}=\left[\begin{array}{ccc}\bar{\Upsilon}_{a b}^{1} & \bar{\Upsilon}_{a b}^{2} & \bar{\Upsilon}_{a b}^{4} \\ * & \bar{\Upsilon}_{a b}^{3} & \bar{\Upsilon}_{a b}^{5} \\ * & * & \bar{\Upsilon}_{a b}^{6}\end{array}\right]$

where $\bar{\Upsilon}_{a b}^{1}=\mathcal{P}\left(\mathcal{A}_{a m}+\mathcal{K}_{b}\right)+\left(\mathcal{A}_{a m}+\mathcal{K}_{b}\right)^{T} \mathcal{P}+\tau^{\alpha} \alpha^{-1} \mathcal{P}+$ $p \mathcal{P}, \bar{\Upsilon}_{a b}^{2}=\mathcal{P} \mathcal{A}_{a m \tau}, \bar{\Upsilon}_{a b}^{3}=-\mathcal{P}, \bar{\Upsilon}_{a b}^{4}=\tau^{\alpha} \alpha^{-1}\left(\mathcal{A}_{a m}+\right.$ $\left.\mathcal{K}_{b}\right)^{T}, \bar{\Upsilon}_{a b}^{5}=\tau^{\alpha} \alpha^{-1} \mathcal{A}_{a m \tau}$, and $\bar{\Upsilon}_{a b}^{6}=-\tau^{\alpha} \alpha^{-1} I$.

Let us consider slack matrices $\overline{\mathcal{U}}=\overline{\mathcal{U}}^{T}$ and $0 \leq \overline{\mathcal{V}}_{a b c}=$ $\overline{\mathcal{V}}_{a b c}^{T}$, then we have

$\left[\sum_{a=1}^{\bar{m}} \sum_{b=1}^{\bar{n}} \sum_{c=1}^{\varrho+1} \nu_{a b c}\left(\underline{\kappa}_{a b c} \underline{\chi}_{a b c}+\bar{\kappa}_{a b c} \bar{\chi}_{a b c}\right)-1\right] \Sigma^{T}(t) \overline{\mathcal{U}} \Sigma(t)=0$,

$-\sum_{a=1}^{\bar{m}} \sum_{b=1}^{\bar{n}} \sum_{c=1}^{\varrho+1} \nu_{a b c}\left(1-\underline{\kappa}_{a b c}\right)\left(\underline{\chi}_{a b c}-\bar{\chi}_{a b c}\right) \Sigma^{T}(t) \overline{\mathcal{V}}_{a b c} \Sigma(t) \geq 0$.

From 21-24, we can get

$$
\begin{aligned}
& D^{\alpha} \mathcal{W}(e(t)) \leq-\int_{t-\tau(t)}^{t}(t-s)^{\alpha-1}\left[\begin{array}{c}
e(t) \\
D^{\alpha}(e(t))
\end{array}\right]^{T} \\
& \times\left[\begin{array}{ll}
\mathcal{P} & 0 \\
0 & I
\end{array}\right]\left[\begin{array}{c}
e(t) \\
D^{\alpha}(e(t))
\end{array}\right] d s \\
& +\sum_{a=1}^{\bar{m}} \sum_{b=1}^{\bar{n}} \sum_{c=1}^{\varrho+1} \nu_{a b c}\left(\underline{\kappa}_{a b c} \underline{\chi}_{a b c}+\bar{\kappa}_{a b c} \bar{\chi}_{a b c}\right) \\
& \times \Sigma^{T}(t) \bar{\Upsilon}_{a b} \Sigma(t)+\left[\sum_{a=1}^{\bar{m}} \sum_{b=1}^{\bar{n}} \sum_{c=1}^{\varrho+1} \nu_{a b c}\right. \\
& \left.\times\left(\underline{\kappa}_{a b c} \underline{\chi}_{a b c}+\bar{\kappa}_{a b c} \bar{\chi}_{a b c}\right)-1\right] \\
& \Sigma^{T}(t) \overline{\mathcal{U}} \Sigma(t)-\sum_{a=1}^{\bar{m}} \sum_{b=1}^{\bar{n}} \sum_{c=1}^{\varrho+1} \nu_{a b c}\left(1-\underline{\kappa}_{a b c}\right) \\
& \times\left(\underline{\chi}_{a b c}-\bar{\chi}_{a b c}\right) \Sigma^{T}(t) \overline{\mathcal{V}}_{a b c} \Sigma(t) \\
& =-\int_{t-\tau(t)}^{t}(t-s)^{\alpha-1}\left[\begin{array}{c}
e(t) \\
D^{\alpha}(e(t))
\end{array}\right]^{T}\left[\begin{array}{ll}
\mathcal{P} & 0 \\
0 & I
\end{array}\right] \\
& \times\left[\begin{array}{c}
e(t) \\
D^{\alpha}(e(t))
\end{array}\right] d s \\
& +\Sigma^{T}(t)\left\{\sum _ { a = 1 } ^ { \overline { m } } \sum _ { b = 1 } ^ { \overline { n } } \sum _ { c = 1 } ^ { \varrho + 1 } \nu _ { a b c } \left(\bar{\kappa}_{a b c} \bar{\Upsilon}_{a b}\right.\right. \\
& \left.-\left(\underline{\chi}_{a b c}-\bar{\chi}_{a b c}\right) \overline{\mathcal{V}}_{a b c}+\bar{\chi}_{a b c} \overline{\mathcal{U}}\right)-\overline{\mathcal{U}} \\
& +\sum_{a=1}^{\bar{m}} \sum_{b=1}^{\bar{n}} \sum_{c=1}^{\varrho+1} \nu_{a b c} \underline{\kappa}_{a b c}\left(\underline{\chi}_{a b c}-\bar{\chi}_{a b c}\right) \\
& \left.\times\left(\bar{\Upsilon}_{a b}+\overline{\mathcal{V}}_{a b c}+\overline{\mathcal{U}}\right)\right\} \Sigma(t) .
\end{aligned}
$$


Now talking $p \rightarrow 1^{+}$in 25, the following inequality holds for some small $h>0$.

$$
\begin{aligned}
D^{\alpha} \mathcal{W}(e(t)) \leq & -h\|e(t)\|^{2}-\int_{t-\tau(t)}^{t}(t-s)^{\alpha-1}\left[\begin{array}{c}
e(t) \\
D^{\alpha}(e(t))
\end{array}\right]^{T} \\
& {\left[\begin{array}{cc}
\mathcal{P} & 0 \\
0 & I
\end{array}\right]\left[\begin{array}{c}
e(t) \\
D^{\alpha}(e(t))
\end{array}\right] d s \leq-h\|e(t)\|^{2} . }
\end{aligned}
$$

Let $\mathcal{P}^{-1}=\overline{\mathcal{P}}, \mathcal{K}_{b} \overline{\mathcal{P}}=\overline{\mathcal{K}}_{b}, \mathcal{P}^{-1} \overline{\mathcal{U}}_{1} \mathcal{P}^{-1}=\mathcal{U}_{1}, \mathcal{P}^{-1} \overline{\mathcal{U}}_{2} \mathcal{P}^{-1}=$ $\mathcal{U}_{2}, \mathcal{P}^{-1} \overline{\mathcal{U}}_{3}=\mathcal{U}_{3}, \mathcal{P}^{-1} \overline{\mathcal{U}}_{4} \mathcal{P}^{-1}=\mathcal{U}_{4}, \mathcal{P}^{-1} \overline{\mathcal{U}}_{5}=\mathcal{U}_{5}, \overline{\mathcal{U}}_{6}=$ $\mathcal{U}_{6}, \mathcal{P}^{-1} \overline{\mathcal{V}}_{1} \mathcal{P}^{-1}=\mathcal{V}_{1}, \mathcal{P}^{-1} \overline{\mathcal{V}}_{2} \mathcal{P}^{-1}=\mathcal{V}_{2}, \mathcal{P}^{-1} \overline{\mathcal{V}}_{3}=\mathcal{U}_{3}$, $\mathcal{P}^{-1} \overline{\mathcal{V}}_{4} \mathcal{P}^{-1}=\mathcal{V}_{4}, \mathcal{P}^{-1} \overline{\mathcal{V}}_{5}=\mathcal{V}_{5}, \overline{\mathcal{V}}_{6}=\mathcal{V}_{6}$. Pre- and postmultiplying (25) with $\operatorname{diag}\{\overline{\mathcal{P}}, \overline{\mathcal{P}}, I\}$, the following inequality holds.

$$
\begin{aligned}
D^{\alpha} \mathcal{W}(e(t)) \leq & \Sigma^{T}(t)\left\{\sum _ { a = 1 } ^ { \overline { m } } \sum _ { b = 1 } ^ { \overline { n } } \sum _ { c = 1 } ^ { \varrho + 1 } \nu _ { a b c } \left(\bar{\kappa}_{a b c} \Upsilon_{a b}\right.\right. \\
& \left.-\left(\underline{\chi}_{a b c}-\bar{\chi}_{a b c}\right) \mathcal{V}_{a b c}+\bar{\chi}_{a b c} \mathcal{U}\right)-\mathcal{U} \\
& +\sum_{a=1}^{\bar{m}} \sum_{b=1}^{\bar{n}} \sum_{c=1}^{\varrho+1} \nu_{a b c} \underline{\kappa}_{a b c}\left(\underline{\chi}_{a b c}-\bar{\chi}_{a b c}\right) \\
& \left.\times\left(\Upsilon_{a b}+\mathcal{V}_{a b c}+\mathcal{U}\right)\right\} \Sigma(t),
\end{aligned}
$$

It follows from [38] that the FO IT2 fuzzy error system (4) is asymptotically stable if $D^{\alpha} \mathcal{W}(e(t))<0$ is satisfied. Thus, it is sufficient to show that $\Upsilon_{a b}+\mathcal{V}_{a b c}+\mathcal{U}>0$ and $\sum_{a=1}^{\bar{m}} \sum_{b=1}^{\bar{n}} \sum_{c=1}^{\varrho+1} \nu_{a b c}\left(\bar{\kappa}_{a b c} \Upsilon_{a b}-\left(\underline{\chi}_{a b c}-\bar{\chi}_{a b c}\right) \mathcal{V}_{a b c}+\bar{\chi}_{a b c} \mathcal{U}\right)-$ $\mathcal{U}<0$. From inequalities (13) and (14), it can be seen that these two conditions are guaranteed. Therefore, it is concluded that the FO IT2 fuzzy error system is asymptotically stable.

Remark 4 It should be mentioned that the developed stability condition for the error system is dependent on the fuzzy MFs of both the plant and the controller. Also, it is noticed that the fuzzy MFs of the plant and controller are chosen as different, which provides more freedom to choose the fuzzy controller than having the same MFs [15]. Thus, both the above-said factors make that the developed theorem has less conservativeness and more freedom to choose the robust feedback controller design for analyzing the required results.

Suppose that the time-varying delay and the nonlinear function are not treated in FO IT2FSs (2) and fuzzy reference model (3). That means, $\rho(t-\tau(t))=0, \rho_{m}(t-\tau(t))=$ 0 and $g(\rho(t))=0$. Then, UDE-based control law 9) can be expressed as follows:

$\vartheta(t)=\sum_{l=1}^{\bar{m}} \sum_{a=1}^{\bar{m}} \sum_{b=1}^{\bar{n}} \lambda_{a}(\rho(t)) \lambda_{a}\left(\rho_{m}(t)\right) \mu_{b}(\rho(t))$

$$
\begin{aligned}
& \times\left\{\mathcal { B } _ { a } ^ { + } \left[( 1 + \frac { 1 } { \mathcal { T } _ { k } } \mathcal { I } ^ { \alpha } ) \left(-\mathcal{K}_{b} e(t)+\mathcal{A}_{a m} \rho(t)\right.\right.\right. \\
& \left.\left.\left.+\mathcal{B}_{a m} r(t)\right)-\mathcal{A}_{l} \rho(t)-\frac{1}{\mathcal{T}_{k}} \rho(t)\right]\right\} .
\end{aligned}
$$

By the support of above controller, the stability conditions for the error system (4) are established without timevarying delay as indicated in the following corollary.

Corollary 1 Consider the FO IT2 fuzzy error system (4) without time-varying delay and the UDE-based controller 28). For the given scalar $\alpha \in(0,1]$, the system (4) without time-varying delay is asymptotically stable, if there exist symmetric positive-definite matrices $\overline{\mathbb{P}} \in \mathbb{R}^{n \times n}, \mathbb{V}_{a b c} \in$ $\mathbb{R}^{n \times n}$, symmetric matrix $\mathbb{U} \in \mathbb{R}^{n \times n},(a \in \mathbb{L}, b \in \mathbb{H}, c \in \mathbb{J})$ and matrices $\mathbb{X}_{b}(b \in \mathbb{H})$ such that

$$
\begin{aligned}
& \Xi_{a b}+\mathbb{V}_{a b c}+\mathbb{U}>0, \forall a, b, c \\
& \sum_{a=1}^{\bar{m}} \sum_{b=1}^{\bar{n}} \sum_{c=1}^{\varrho+1}\left[\bar{\varepsilon}_{a b a_{1} a_{2} \ldots a_{n} v c} \Xi_{a b}-\left(\underline{\varepsilon}_{a b a_{1} a_{2} \ldots a_{n} v c}\right.\right. \\
& \left.\left.-\bar{\varepsilon}_{a b a_{1} a_{2} \ldots a_{n} v c}\right) \mathbb{V}_{a b c}+\bar{\varepsilon}_{a b a_{1} a_{2} \ldots a_{n} v c} \mathbb{U}\right]-\mathbb{U}<0, \forall v, c,
\end{aligned}
$$

where $\Xi_{a b}=\mathcal{A}_{a m}^{T} \overline{\mathbb{P}}+\overline{\mathbb{P}} \mathcal{A}_{a m}+\mathbb{X}_{b}+\mathbb{X}_{b}^{T}$. Then, the controller gain matrices can be expressed as $\mathcal{K}_{b}=\overline{\mathbb{P}}^{-1} \mathbb{X}_{b}$.

In the upcoming section, we will show the effectiveness of the proposed results in three numerical examples. In particular, to reduce the computational complexity, the upper and lower MFs (10) and (11) with state space $j=1$ (i.e. $\rho_{1}(t)$ ), sub-FOU $c=1$ and sub domain $a_{1}=1,2$ are considered in both examples.

\section{Numerical simulations}

In this section, four numerical examples with simulations are presented to illustrate the validity of the proposed UDEbased control scheme. Specifically, Example 1 exhibits the efficiency of the proposed method against parameter uncertainty and external disturbance. Next, in the practical example, a permanent magnet synchronous motor [40,41] is considered and validated in Example 2. Example 3 discusses the selection filter parameter to estimate the lumped unknown disturbance effect in the designed UDE-based controller. Example 4 provides the practical prospects of the desired control methods for FO Rössler fuzzy systems.

Example 1 Consider the FO IT2FSs in the form of (2) with four fuzzy rules and the following system matrices:

$\mathcal{A}_{1}=\left[\begin{array}{cc}-1 & 0 \\ 1 & -1\end{array}\right], \mathcal{A}_{2}=\left[\begin{array}{ll}0 & -1 \\ 0 & -3\end{array}\right], \mathcal{A}_{3}=\left[\begin{array}{cc}-1 & -2 \\ 1 & -1\end{array}\right]$, 
$\mathcal{A}_{4}=\left[\begin{array}{cc}-2 & 1 \\ 0 & -1\end{array}\right], \mathcal{A}_{1 \tau}=\left[\begin{array}{ll}1 & 0.5 \\ 1 & 0.6\end{array}\right], \mathcal{A}_{2 \tau}=\left[\begin{array}{ll}0.5 & 0.1 \\ 0.2 & 0.3\end{array}\right]$,

$\mathcal{A}_{3 \tau}=\left[\begin{array}{cc}0 & 0.2 \\ 0.1 & 0.1\end{array}\right], \mathcal{A}_{4 \tau}=\left[\begin{array}{ll}0.7 & 0.2 \\ 0.5 & 0.4\end{array}\right], \mathcal{C}_{1}=\left[\begin{array}{cc}1 & 1 \\ 0.5 & -1\end{array}\right]$,

$\mathcal{C}_{2}=\left[\begin{array}{ll}0.5 & 1 \\ 1.5 & 1\end{array}\right], \mathcal{C}_{3}=\left[\begin{array}{cc}0 & -1 \\ 0 & 2\end{array}\right]$, and $\mathcal{C}_{4}=\left[\begin{array}{cc}1 & 1 \\ -1 & -1\end{array}\right]$.

Further, the system matrices of reference model (3) with four fuzzy rules $(l=1,2,3,4)$ are chosen as follows:

$\mathcal{A}_{1 m}=\left[\begin{array}{cc}0 & 1 \\ 0 & -2\end{array}\right], \mathcal{A}_{2 m}=\left[\begin{array}{ll}1 & -1 \\ 2 & -1\end{array}\right], \mathcal{A}_{3 m}=\left[\begin{array}{cc}-2 & 1 \\ -2 & -1\end{array}\right]$

$\mathcal{A}_{4 m}=\left[\begin{array}{ll}-1 & -2 \\ -1 & -3\end{array}\right], \mathcal{A}_{1 m \tau}=\left[\begin{array}{cc}1 & 1 \\ 0 & -3\end{array}\right], \mathcal{A}_{2 m \tau}=\left[\begin{array}{cc}0 & -2 \\ 0 & 1\end{array}\right]$,

$\mathcal{A}_{3 m \tau}=\left[\begin{array}{cc}0 & 1 \\ 0 & -2\end{array}\right], \mathcal{A}_{4 m \tau}=\left[\begin{array}{cc}-1 & 0 \\ 1 & -2\end{array}\right]$,

and $\mathcal{B}_{l}=\mathcal{B}_{l m}=I_{2 \times 2},(l=1,2,3,4)$.

Table 1 Plants membership functions

\begin{tabular}{l|c}
\hline \hline \multicolumn{1}{c|}{ UBs } & LBs \\
\hline \hline $\bar{\lambda}_{\mathbb{E}_{1}^{1}}\left(\rho_{1}\right)=1-0.23 e^{-\frac{\rho_{1}^{2}}{0.25}}$ & $\underline{\underline{E}}_{1}\left(\rho_{1}\right)=1-e^{-\frac{\rho_{1}^{2}}{1.2}}$ \\
$\bar{\lambda}_{\mathbb{E}_{1}^{2}}\left(\rho_{1}\right)=1-0.23 e^{-\frac{\rho_{1}^{2}}{0.25}}$ & $\underline{\lambda}_{\mathbb{E}_{1}^{2}}\left(\rho_{1}\right)=1-e^{-\frac{\rho_{1}^{2}}{1.2}}$ \\
$\bar{\lambda}_{\mathbb{E}_{1}^{3}}\left(\rho_{1}\right)=e^{-\frac{\rho_{1}^{2}}{1.2}}$ & $\underline{\lambda}_{\mathbb{E}_{1}^{3}}\left(\rho_{1}\right)=0.23 e^{-\frac{\rho_{1}^{2}}{0.25}}$ \\
$\bar{\lambda}_{\mathbb{E}_{1}^{4}}\left(\rho_{1}\right)=e^{-\frac{\rho_{1}^{2}}{1.2}}$ & $\underline{\lambda}_{\mathbb{E}_{1}^{4}}\left(\rho_{1}\right)=0.23 e^{-\frac{\rho_{1}^{2}}{0.25}}$ \\
$\bar{\lambda}_{\mathbb{E}_{2}^{1}}\left(\rho_{1}\right)=e^{-\frac{\rho_{1}^{2}}{1.5}}$ & $\underline{\lambda}_{\mathbb{E}_{2}^{1}}\left(\rho_{1}\right)=0.5 e^{-\frac{\rho_{1}^{2}}{0.25}}$ \\
$\bar{\lambda}_{\mathbb{E}_{2}^{2}}\left(\rho_{1}\right)=1-0.5 e^{-\frac{\rho_{1}^{2}}{0.25}}$ & $\underline{\lambda}_{\mathbb{E}_{2}^{2}}\left(\rho_{1}\right)=1-e^{-\frac{\rho_{1}^{2}}{1.5}}$ \\
$\bar{\lambda}_{\mathbb{E}_{2}^{3}}\left(\rho_{1}\right)=e^{-\frac{\rho_{1}^{2}}{1.5}}$ & $\underline{\lambda}_{\mathbb{E}_{2}^{3}}\left(\rho_{1}\right)=0.5 e^{-\frac{\rho_{1}^{2}}{0.25}}$ \\
$\bar{\lambda}_{\mathbb{E}_{2}^{4}}\left(\rho_{1}\right)=1-0.5 e^{-\frac{\rho_{1}^{2}}{0.25}}$ & $\underline{\lambda}_{\mathbb{E}_{2}^{4}}\left(\rho_{1}\right)=1-e^{-\frac{\rho_{1}^{2}}{1.5}}$ \\
\hline \hline
\end{tabular}

Table 2 Controllers membership functions

\begin{tabular}{l|c}
\hline \hline \multicolumn{1}{c|}{ UBs } & LBs \\
\hline \hline $\bar{\mu}_{\mathbb{G}_{1}^{1}}\left(\rho_{1}\right)=e^{-\rho_{1}^{2}}$ & $\underline{\mu}_{\mathbb{G}_{1}^{1}}\left(\rho_{1}\right)=e^{\frac{-\rho_{1}^{2}}{0.5}}$ \\
$\bar{\mu}_{\mathbb{G}_{1}^{2}}\left(\rho_{1}\right)=1-e^{\frac{-\rho_{1}^{2}}{0.5}}$ & $\underline{\mu}_{\mathbb{G}_{1}^{2}}\left(\rho_{1}\right)=1-e^{-\rho_{1}^{2}}$ \\
\hline \hline
\end{tabular}

Further, the reference command input and time delay are chosen as $\left[r_{1}(t) r_{2}(t)\right]^{T}=\left[\begin{array}{lll}1.2 \sin (\pi t) & 1.2 \cos (\pi t)\end{array}\right]^{T}$ and $\tau(t)=0.1 \cos (t)$, respectively.

Moreover, the upper bounds (UBs) and lower bounds (LBs) of four-rule MFs for the considered fuzzy model (2) and two fuzzy-rule MFs of the controller (5) are given in Table 1 and Table 2, respectively. In which, the weight coefficient functions of the plant and the controller are taken as $\underline{y}_{1}(\rho(t))=\underline{y}_{4}(\rho(t))=\cos ^{2}(\rho(t)), \underline{y}_{2}(\rho(t))=\underline{y}_{3}(\rho(t))=$ $\sin ^{2}(\rho(t)), \underline{z}_{b}(\rho(t))=\sin ^{2}(\rho(t)), \bar{y}_{l}(\rho(t))=1-\underline{y}_{l}(\rho(t))$ and $\bar{z}_{b}(\rho(t))=1-\underline{z}_{b}(\rho(t))(l=1,2,3,4$, and $b=1,2)$ respectively. The corresponding MFs of considered system and proposed controller are plotted in Figs. 2 and 3 respectively. In addition, to improve the accuracy of the fuzzy model the system state $\rho_{1} \in[-\pi / 3, \pi / 3]$ is divided to 500 equal-size sub-states. To express the lower and upper MFs as $w_{11 v}\left(\rho_{1}\right)=1-\frac{\rho_{1}-\underline{\rho}_{1, v}}{\bar{\rho}_{1, v}-\underline{\rho}_{1, v}}$ and $w_{12 v}\left(\rho_{1}\right)=1-w_{11 v}\left(\rho_{1}\right)$, where $\underline{\rho}_{1, v}=\frac{2 \pi}{1500}(v-251)$ and $\bar{\rho}_{1, v}=\frac{2 \pi}{1500}(v-250)$ $(v=1,2, \ldots, 500)$ and the corresponding constant scalars can be determined as $\bar{\varepsilon}_{a b 1 v}=\bar{\lambda}_{a}\left(\underline{\rho}_{1, v}\right) \bar{\mu}_{b}\left(\underline{\rho}_{1, v}\right), \underline{\varepsilon}_{a b 1 v}=$ $\underline{\lambda}_{a}\left(\underline{\rho}_{1, v}\right) \underline{\mu}_{b}\left(\underline{\rho}_{1, v}\right), \bar{\varepsilon}_{a b 2 v}=\bar{\lambda}_{a}\left(\bar{\rho}_{1, v}\right) \bar{\mu}_{b}\left(\bar{\rho}_{1, v}\right)$ and $\underline{\varepsilon}_{a b 2 v}=$ $\underline{\lambda}_{a}\left(\bar{\rho}_{1, v}\right) \underline{\mu}_{b}\left(\bar{\rho}_{1, v}\right)$ for all $v$. Let $\mathcal{T}_{k}=0.001, \tau=0.1$, under these parameters by solving the linear matrix inequality (LMI) constraints developed in Theorem 1 1 with the aid of LMI toolbox [39], the control gain matrices are obtained as

$\mathcal{K}_{1}=\left[\begin{array}{cc}-64.4571 & 76.0176 \\ 77.0147 & -139.2830\end{array}\right]$,
$\mathcal{K}_{2}=\left[\begin{array}{cc}-74.9535 & 88.7277 \\ 88.1766 & -159.1919\end{array}\right]$.

Then, for simulation purpose, unknown disturbance in the dynamics of system (2) are taken as follows:

$$
\begin{aligned}
& \beta_{1}(t)=\left\{\begin{array}{lc}
10 \sin (\pi t), & 3 \leq t \leq 7 \\
3, & 7<t \leq 10 \\
5 \cos (\pi t), & 10<t \leq 18 \\
0, & \text { otherwise. }
\end{array}\right. \text { and } \\
& \beta_{2}(t)=\left\{\begin{array}{lc}
7 \cos (\pi t), & 3 \leq t \leq 7 \\
6, & 7<t \leq 10 \\
3 \sin (\pi t), & 10<t \leq 16 \\
0, & \text { otherwise. }
\end{array}\right.
\end{aligned}
$$

Let the nonlinear functions $g(\rho(t))=\left[g_{1}(\rho(t)) g_{2}(\rho(t))\right]^{T}$ and the unknown uncertainty $\Delta \mathcal{A}_{l}(t)$ be given by

$$
\begin{aligned}
& g_{1}(\rho(t))=3 \sin \left(\rho_{1}(t)\right) \cos \left(\rho_{2}(t)\right) \\
& g_{2}(\rho(t))=\sin \left(\rho_{2}(t)\right) \cos \left(\rho_{1}(t)\right) \\
& \Delta \mathcal{A}_{1}(t)=\Delta \mathcal{A}_{3}(t)=\left[\begin{array}{ll}
0.3 \sin (t) & 0.2 \cos (t) \\
0.2 \cos (t) & 0.4 \sin (t)
\end{array}\right], \text { and } \\
& \Delta \mathcal{A}_{2}(t)=\Delta \mathcal{A}_{4}(t)=\left[\begin{array}{ll}
0.3 \cos (t) & 0.1 \sin (t) \\
0.1 \sin (t) & 0.6 \cos (t)
\end{array}\right]
\end{aligned}
$$

Let us take the initial states as $\rho_{1}(0)=-1, \rho_{2}(0)=1$, $\rho_{1 m}(0)=0.2$, and $\rho_{2 m}(0)=0.5$ and fractional-derivative order $\alpha=0.7$. Under these initial conditions, the simulation results are provided to show the efficiency of the obtained controller.

The state responses of the FO IT2FSs (2) are presented in Figs. 4 and 5, respectively. From these figures, 'it can be concluded that the proposed controller can guarantee that 

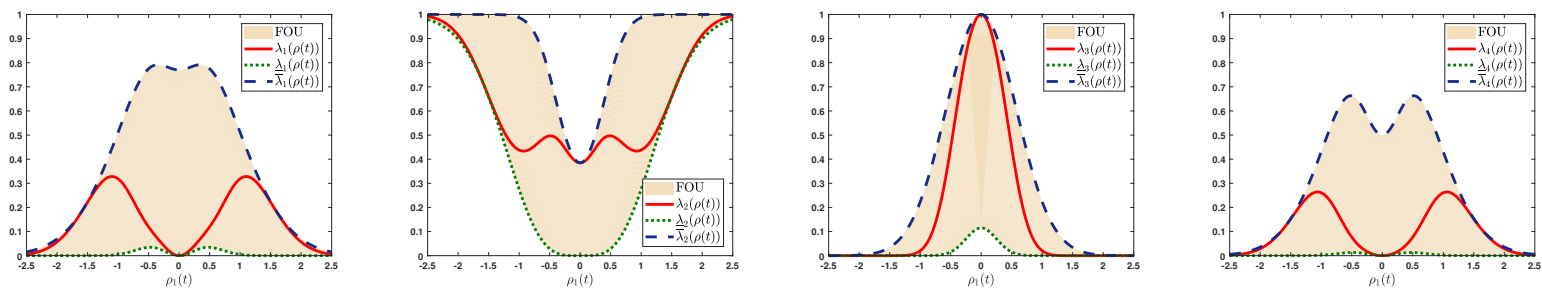

Fig. 2 Membership functions of plant $\lambda_{l}, l=1,2,3,4$
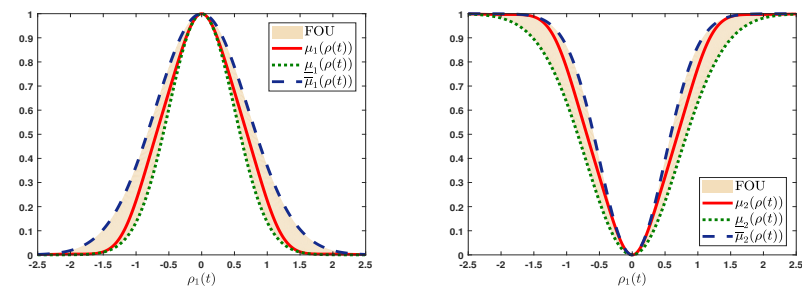

Fig. 3 Membership functions of controller $\mu_{b}, b=1,2$
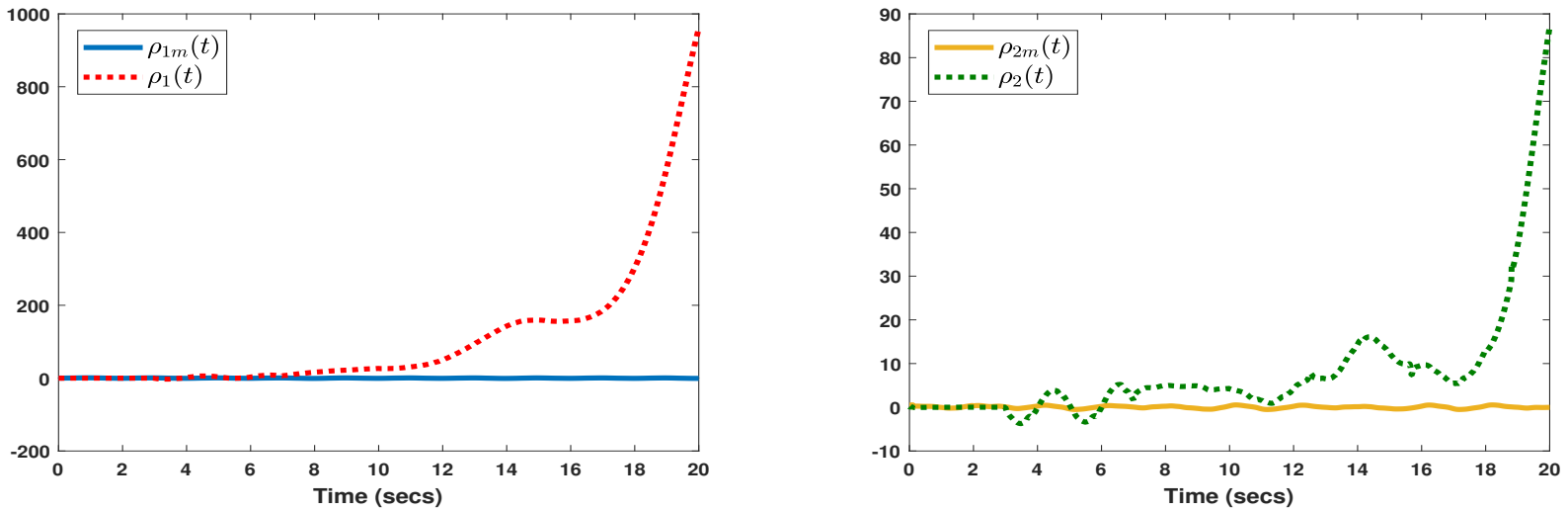

Fig. 4 State responses of the open-loop system
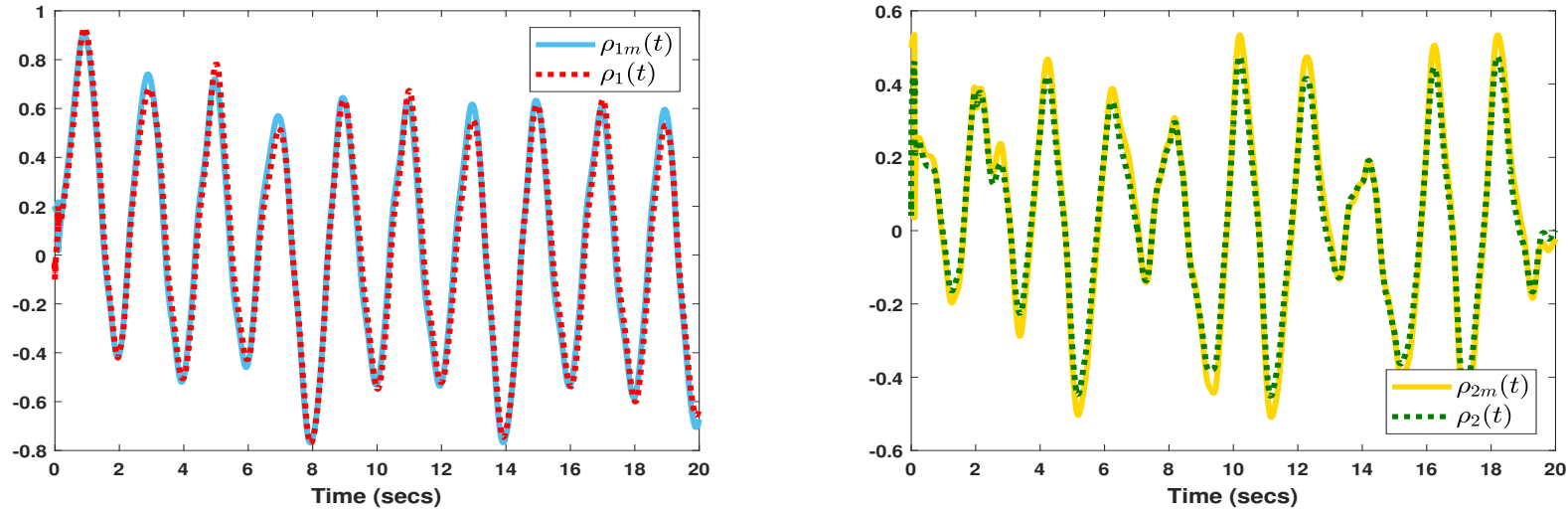

Fig. 5 State responses of the closed-loop system

the error signals asymptotically converge to zero and that the effect of the lumped unknown nonlineartiy is estimated effectively. In the absence of controller, the system states fails to track the reference signal. Moreover, corresponding control performance is shown in Fig. 6. Fig. 7 displays the lumped unknown nonlinearity and their estimations. Thus, from these simulation results, it is easy to conclude that the proposed controller guarantees that the error responses are asymptotically converged to zero and also perfectly estimates the effect of lumped unknown nonlinearity effectively. 


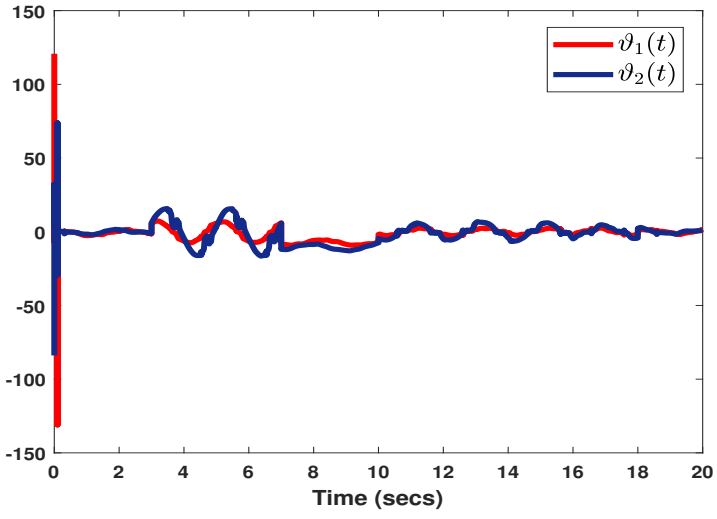

Fig. 6 Control responses

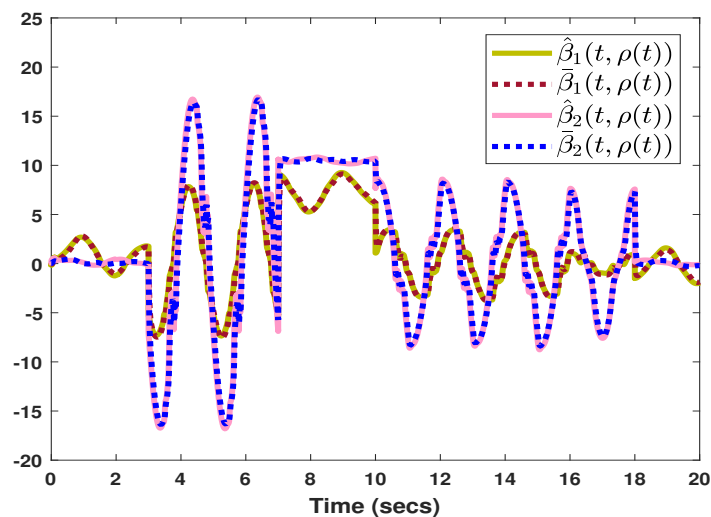

Fig. 7 Lumped nonlinearity and its estimation

Remark 5 It should be mentioned that in the simulations, the control signal is passed through the input channel along with the uncertainty and external disturbances. To identify and reduce the effect of uncertainty and external disturbances, the proposed control should take more effort which is the reason that the control signals reach peak values at the beginning of the simulations. Moreover, due to the uncertainty and external disturbances effect on the control system, the control signals reach peak values at the beginning in a very short period. It can be observed from Fig. 6 that though the control signals initially reach very high value, after some short period the signals converge to zero very quickly which shows the effectiveness of the designed controller.

Example 2 In practical example, the permanent magnet synchronous motor [40] is considered. It is considered that there happens to be a time delay in this system, which is characterized as:

$$
\begin{aligned}
t_{0} \mathcal{D}_{t}^{0.9} \rho(t) & =\sum_{l=1}^{2} \lambda_{l}(\rho(t))\left[\left((1-a) \mathcal{A}_{l}+\Delta \mathcal{A}_{l}(t)\right) \rho(t)\right. \\
& \left.+a \mathcal{A}_{l} \rho(t-\tau(t))+\mathcal{B}_{l} \vartheta(t)+\mathcal{C}_{l} \beta(t)\right]+g(\rho(t)),
\end{aligned}
$$

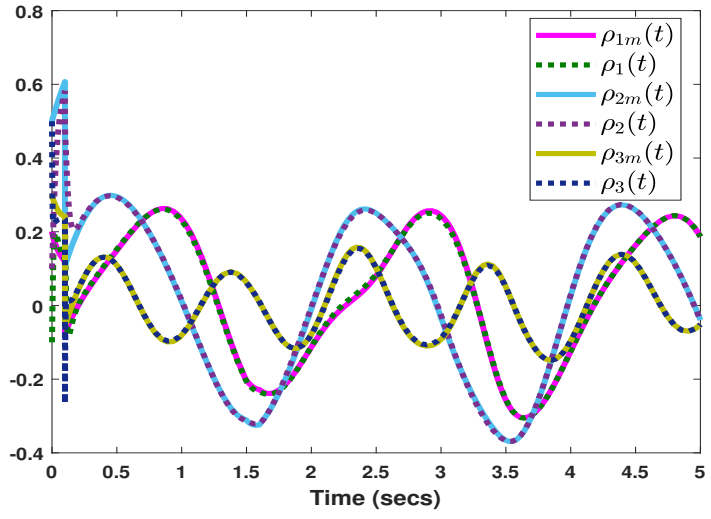

Fig. 8 State responses of the closed-loop system

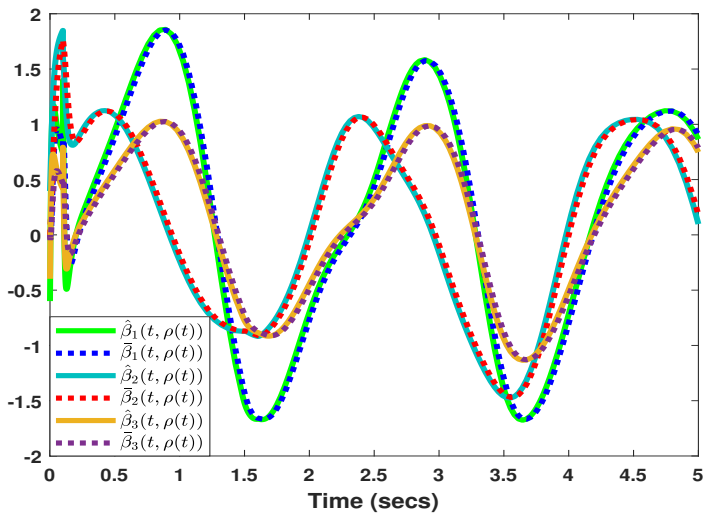

Fig. 9 Lumped nonlinearity and its estimation

where

$$
\begin{aligned}
& \mathcal{A}_{1}=\left[\begin{array}{ccc}
-d_{1} & 0 & d_{1} \\
0 & -1 & d_{3} \\
d_{2} & -d_{3} & -1
\end{array}\right], \mathcal{A}_{2}=\left[\begin{array}{ccc}
-d_{1} & 0 & d_{1} \\
0 & -1 & r_{4} \\
d_{2} & -d_{4} & -1
\end{array}\right], \\
& \mathcal{B}_{l}=\left[\begin{array}{lll}
1 & 0 & 0 \\
0 & 1 & 0 \\
0 & 0 & 1
\end{array}\right], \mathcal{C}_{1}=\left[\begin{array}{l}
1 \\
0 \\
1
\end{array}\right], \mathcal{C}_{2}=\left[\begin{array}{c}
1 \\
0 \\
-1
\end{array}\right], g_{1}(\rho(t))=3 \sin \left(\rho_{1}(t)\right), \\
& g_{2}(\rho(t))=\sin \left(2 \rho_{2}(t)\right), g_{3}(\rho(t))=2 \sin \left(\rho_{1}(t)\right) \cos \left(\rho_{2}(t)\right), \\
& \Delta \mathcal{A}_{l}(t)=\left[\begin{array}{ccc}
1.5 \sin (t) & 0 & -1.5 \sin (t) \\
-1.5 \sin (t) & 0 & 1.5 \sin (t) \\
0 & 0 & 0
\end{array}\right] .
\end{aligned}
$$

with $a=0.2, d_{1}=-1.9 d_{2}=5, d_{3}=-1.5$ and $d_{4}=3$. The upper and lower MFs of the IT2FSs are considered in [41].

The parameters of the reference model are defined as follows:

$\mathcal{A}_{1 m}=\left[\begin{array}{ccc}-3 & 0 & -2 \\ 0 & 1 & -1 \\ 0 & 1 & -5\end{array}\right], \mathcal{A}_{2 m}=\left[\begin{array}{ccc}-2 & 1 & -5 \\ 1 & -1 & -2 \\ -1 & 0 & -4\end{array}\right]$ 
$\mathcal{A}_{1 m \tau}=\left[\begin{array}{ccc}-1 & 1 & 0 \\ 0 & 1 & -2 \\ 1 & 1 & -1\end{array}\right], \mathcal{A}_{2 m \tau}=\left[\begin{array}{ccc}-1 & 1 & -1 \\ 0 & -2 & 1 \\ -1 & 0 & -2\end{array}\right]$,

$\mathcal{B}_{l m}=\left[\begin{array}{lll}1 & 0 & 0 \\ 0 & 1 & 0 \\ 0 & 0 & 1\end{array}\right]$

The reference input, initial states, time delay, disturbance and simulation parameters are defined below:

$\left[r_{1}(t), r_{2}(t), r_{3}(t)\right]^{T}=[1.2 \sin (\pi t), 1.2 \cos (\pi t), \sin (2 \pi t)]^{T}$,

$\left[\rho_{1}(0), \rho_{2}(0), \rho_{3}(0)\right]^{T}=[-0.1,0.1,0.5]^{T}$,

$\left[\rho_{1 m}(0), \rho_{2 m}(0), \rho_{3 m}(0)\right]^{T}=[0.2,0.5,0.3]^{T}, \tau=0.1$,

$\tau(t)=0.1 \cos (t), \beta(t)=\sin (t) e^{-2 t}, \alpha=0.9, \mathcal{T}_{k}=0.01$.

Based on the conditions obtained in Theorem 1 with the above mentioned parameters, a set of controller gain values are obtained as

$\begin{aligned} \mathcal{K}_{1}= & {\left[\begin{array}{ccc}-63.3648 & 0.0270 & -58.9855 \\ 0.2616 & -33.4233 & 14.5663 \\ -58.8226 & 14.1586 & -159.9604\end{array}\right], } \\ \mathcal{K}_{2}= & {\left[\begin{array}{ccc}-50.0185 & 0.1707 & -46.5004 \\ 0.5108 & -26.5024 & 11.8139 \\ -46.3260 & 11.1638 & -126.1175\end{array}\right] . }\end{aligned}$

Fig. 8 plots the state responses under the control scheme (9), where it can be seen that the proposed controller satisfies the required estimation constraints. The actual lumped nonlinearity acting on the system and its estimation are displayed in Fig. 9. In particular, the designed UDE-based dynamics are perfectly estimated and compensated tracking performance within a very short period. Hence, the proposed controller performs successfully.

Remark 6 The time-delay control estimation technique is proposed in [40], which is based on the assumption that a continuous signal remains unchanged during a small enough period. By using the past observation of uncertainties and disturbances, the control action is modified directly, instead of adjusting controller gains, or identifying system parameters. However, it suffers from some problems, which are caused by the need for the derivatives of system states and the difficulty in stability analysis due to the use of timedelayed signals.

Example 3 Consider the FO IT2FSs with three fuzzy rules in the following form:

$$
{ }_{t_{0}} \mathcal{D}_{t}^{\alpha} \rho(t)=\sum_{l=1}^{3} \lambda_{l}\left\{\left(\mathcal{A}_{l}+\Delta \mathcal{A}_{l}(t)\right) \rho(t)+\mathcal{B}_{l} \vartheta(t)\right\}
$$

where

$$
\begin{aligned}
& \mathcal{A}_{1}=\left[\begin{array}{ccc}
1 & 2 & -1 \\
-2 & 1 & 1 \\
1 & 0 & -1
\end{array}\right], \mathcal{A}_{2}=\left[\begin{array}{ccc}
-1 & 0 & 1 \\
-1 & 1 & -1 \\
1 & 1 & -2
\end{array}\right], \mathcal{A}_{3}=\left[\begin{array}{ccc}
1 & 1 & -2 \\
1 & -1 & -2 \\
-1 & 0 & 1
\end{array}\right], \\
& \mathcal{B}_{l}=\left[\begin{array}{lll}
1 & 0 & 0 \\
0 & 1 & 0 \\
0 & 0 & 1
\end{array}\right], \Delta \mathcal{A}_{1}(t)=\left[\begin{array}{ccc}
5 \sin (t) & \cos (\pi t) & 3 \sin (t) \\
\sin (t) & \cos (\pi t) & 0.1 \sin (\pi t) \\
5 \sin (t) & 2 \cos (\pi t) & \sin (\pi t)
\end{array}\right], \\
& \Delta \mathcal{A}_{2}(t)=\left[\begin{array}{ccc}
\sin (\pi t) & 0.6 \cos (\pi t) & 0.8 \cos (t) \\
0.3 \sin (\pi t) & 0.7 \sin (t) & 0.2 \sin (\pi t) \\
\cos (\pi t) & 0.5 \cos (\pi t) & 0.9 \cos (t)
\end{array}\right], \\
& \text { and } \Delta \mathcal{A}_{3}(t)=\left[\begin{array}{ccc}
0.1 \cos (t) & 0.7 \sin (\pi t) & \cos (t) \\
0.6 \cos (t) & 0.9 \sin (\pi t) & 0.9 \cos (\pi t) \\
\cos (t) & \sin (t) & \sin (\pi t)
\end{array}\right] .
\end{aligned}
$$

\begin{tabular}{|c|c|}
\hline UBs & LBs \\
\hline $\bar{\lambda}_{\mathbb{E}_{1}^{1}}\left(\rho_{1}\right)=1-0.23 e^{-\frac{\rho_{1}^{2}}{0.3}}$ & $\underline{\lambda}_{\mathbb{E}_{1}^{1}}\left(\rho_{1}\right)=1-e^{-\frac{\rho_{1}^{2}}{1.5}}$ \\
\hline $\bar{\lambda}_{\mathbb{E}_{1}^{2}}\left(\rho_{1}\right)=1-0.23 e^{-\frac{\rho_{1}^{2}}{0.3}}$ & $\underline{\lambda}_{\mathbb{E}_{1}^{2}}\left(\rho_{1}\right)=1-e^{-\frac{\rho_{1}^{2}}{1.5}}$ \\
\hline $\bar{\lambda}_{\mathbb{E}_{1}^{3}}\left(\rho_{1}\right)=e^{-\frac{\rho_{1}^{2}}{1.5}}$ & $\underline{\lambda}_{\mathbb{E}_{1}^{3}}\left(\rho_{1}\right)=0.23 e^{-\frac{\rho_{1}^{2}}{0.3}}$ \\
\hline $\bar{\lambda}_{\mathbb{E}_{2}^{1}}\left(\rho_{1}\right)=e^{-\frac{\rho_{1}^{2}}{2.5}}$ & $\underline{\lambda}_{\mathbb{E}_{2}^{1}}\left(\rho_{1}\right)=0.5 e^{-\frac{\rho_{1}^{2}}{0.2}}$ \\
\hline $\bar{\lambda}_{\mathbb{E}_{2}^{2}}\left(\rho_{1}\right)=1-0.5 e^{-\frac{\rho_{1}^{2}}{0.2}}$ & $\underline{\lambda}_{\mathbb{E}_{2}^{2}}\left(\rho_{1}\right)=1-e^{-\frac{\rho_{1}^{2}}{2.5}}$ \\
\hline $\bar{\lambda}_{\mathbb{E}_{2}^{3}}\left(\rho_{1}\right)=e^{-\frac{\rho_{1}^{1}}{2.5}}$ & $\underline{\lambda}_{\mathbb{E}_{2}^{3}}\left(\rho_{1}\right)=0.5 e^{-\frac{\rho_{1}^{2}}{0.2}}$ \\
\hline
\end{tabular}

Table 3 Plants membership functions

Further, the parameters of the reference model of the form (5) are selected as follows:

$$
\begin{aligned}
& \mathcal{A}_{1 m}=\left[\begin{array}{ccc}
0 & 5 & 10 \\
12 & 3 & 10 \\
1 & 5 & -5
\end{array}\right], \mathcal{A}_{2 m}=\left[\begin{array}{ccc}
-1 & 4 & -3 \\
2 & -6 & -7 \\
5 & 1 & -6
\end{array}\right], \\
& \mathcal{A}_{3 m}=\left[\begin{array}{ccc}
0 & -5 & 7 \\
0 & 10 & -3 \\
0 & -2 & -7
\end{array}\right], \mathcal{B}_{a m}=\left[\begin{array}{lll}
1 & 0 & 0 \\
0 & 1 & 0 \\
0 & 0 & 1
\end{array}\right] .
\end{aligned}
$$

with the reference input

$$
r(t)=[1.2 \sin (\pi t) 1.2 \cos (\pi t) \sin (2 \pi t)]^{T} .
$$

The upper and lower MFs of the system (31) are presented in Table 3 In addition, the upper and lower bounds of MFs of the controller are taken same as the MFs of the Example 1 as in Table 2 Moreover, we consider $\rho_{1} \in[-100,100]$ and is split into 1000 equal-size sub-states. Let us choose the initial states as $\rho_{1}(0)=1, \rho_{2}(0)=2, \rho_{3}(0)=3$, $\rho_{1 m}(0)=0.2, \rho_{2 m}(0)=0.6, \rho_{3 m}(0)=0.1$ and fractionalderivative order $\alpha=0.7$. From the feasible solutions of LMIs in Corollary 1, we can get the feedback control gain matrices as follows:

$\mathcal{K}_{1}=\left[\begin{array}{ccc}-7.6194 & -6.3806 & -11.1873 \\ 10.8845 & -63.4957 & 1.6564 \\ -7.6432 & 9.0576 & -11.2766\end{array}\right]$, 


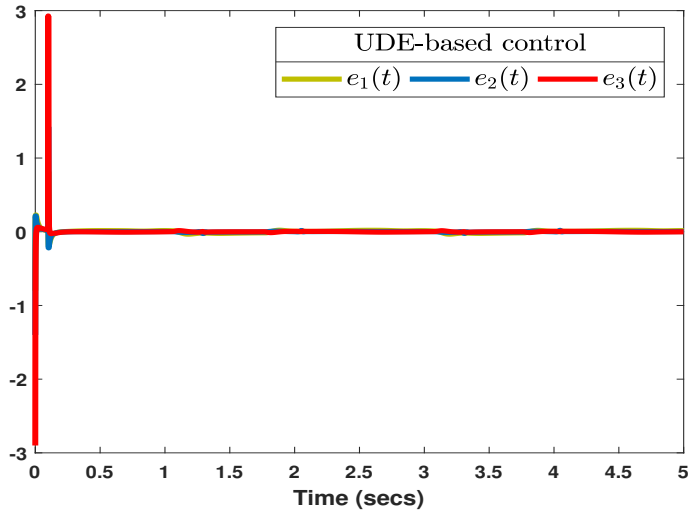

Fig. 10 Error responses for closed-loop system

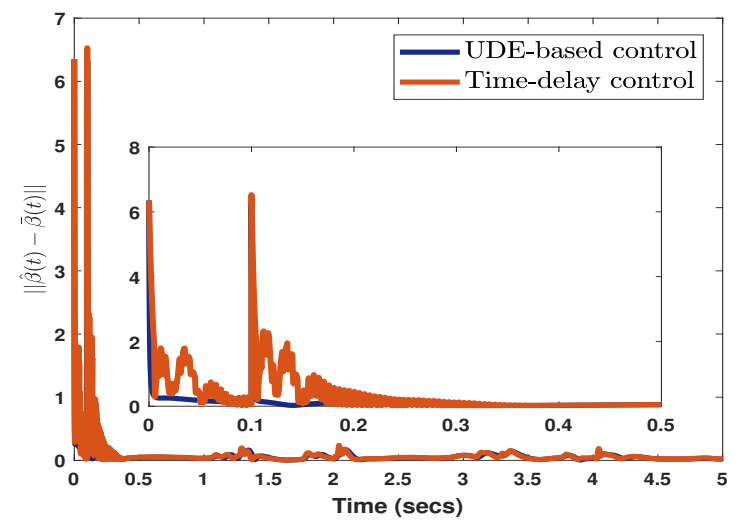

Fig. 11 Lumped uncertainty and its estimation

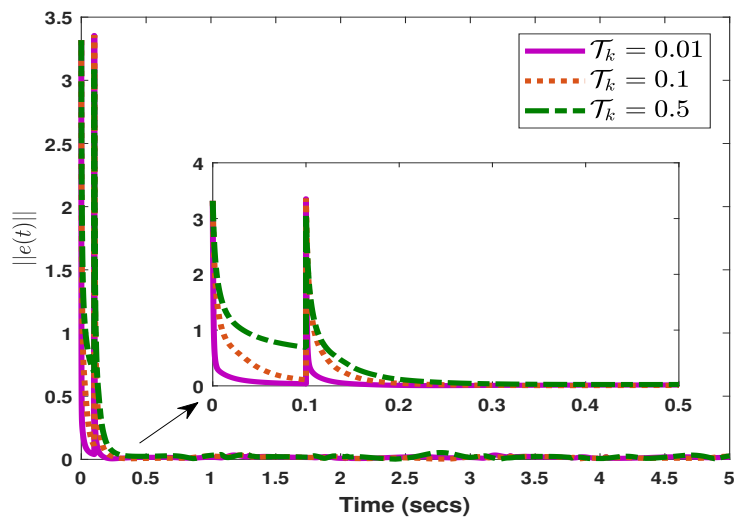

Fig. 12 Tracking error for various bandwidth

$\mathcal{K}_{2}=\left[\begin{array}{ccc}-8.5863 & -6.0351 & -11.7922 \\ 10.8306 & -63.5511 & 1.8458 \\ -8.0549 & 10.0444 & -11.9460\end{array}\right]$

In order to compare with time-delay control, the following time-delay control design is provided in [42]:

$\vartheta(t)=\vartheta(t-L)+\sum_{l=1}^{\bar{m}} \sum_{a=1}^{\bar{m}} \sum_{b=1}^{\bar{n}} \lambda_{l}(\rho(t)) \lambda_{a}\left(\rho_{m}(t)\right) \mu_{b}\left(\rho_{m}(t)\right)$

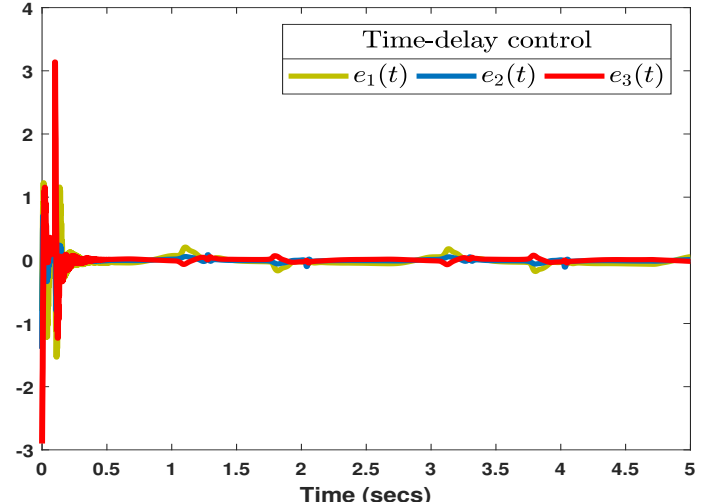

$$
\begin{aligned}
& \times \mathcal{B}_{l}^{+}\left[-\mathcal{K}_{b} e(t)+\mathcal{A}_{a m} \rho(t)+\mathcal{B}_{a m} r(t)-\mathcal{A}_{l} \rho(t)\right. \\
& \left.-\frac{\dot{\rho}(t)-\dot{\rho}(t-L)}{L}\right]
\end{aligned}
$$

where $L=0.005 \mathrm{~s}$ and the derivative of $\dot{\rho}(t)$ is approximated by $\frac{\dot{\rho}(t)-\dot{\rho}(t-L)}{L}$.

Based on UDE-based control design 28 and time-delay control design 32, the simulation result for the controlled error signals and lumped uncertainty estimations are presented in Figs. 10 and 11. That is, it can be seen that the state of the system can track the reference state under the proposed controller and time-delay controller, which verifies the developed theoretical result in Corollary 1 In particular, there are oscillations in the error signals and lumped uncertainty estimations for time-delay control but in the case with UDE-based control, the control signal is very smooth. Moreover, Fig. 12 depicts that the tracking error performance of the closed-loop system under various cut-off frequency of the filter. It is easy to conclude that when the filter parameter decreases then both tracking performance and disturbance estimation performance improve. Moreover, $\mathcal{T}_{k}$ is the inverse of the cut-off frequency and it is well-known that high frequency signals highly affect the stability margin. So, it is important to select the filter parameter for trade-off between the stability margin and system performance against the unknown lumped uncertainty.

Example 4 Let us consider the FO Rössler system [22] as follows:

$\left\{\begin{array}{l}d^{\alpha} \rho_{1}(t) / d t^{\alpha}=-\rho_{2}(t)-\rho_{3}(t) \\ d^{\alpha} \rho_{2}(t) / d t^{\alpha}=\rho_{1}(t)+s_{1} \rho_{3}(t) \\ d^{\alpha} \rho_{3}(t) / d t^{\alpha}=s_{2} \rho_{1}(t)-\left(s_{3}-\rho_{1}(t)\right) \rho_{3}(t)+\vartheta(t)+\beta(t)\end{array}\right.$

where $\rho_{1}(t) \in\left[s_{3}-s_{4}, s_{3}+s_{4}\right]$. The above system can be represented with two fuzzy rules in the following form;

${ }_{t_{0}} \mathcal{D}_{t}^{\alpha} \rho(t)=\sum_{l=1}^{2} \lambda_{l}\left\{\mathcal{A}_{l} \rho(t)+\mathcal{B}_{l} \vartheta(t)+\mathcal{C}_{l} \beta(t)\right\}$, 


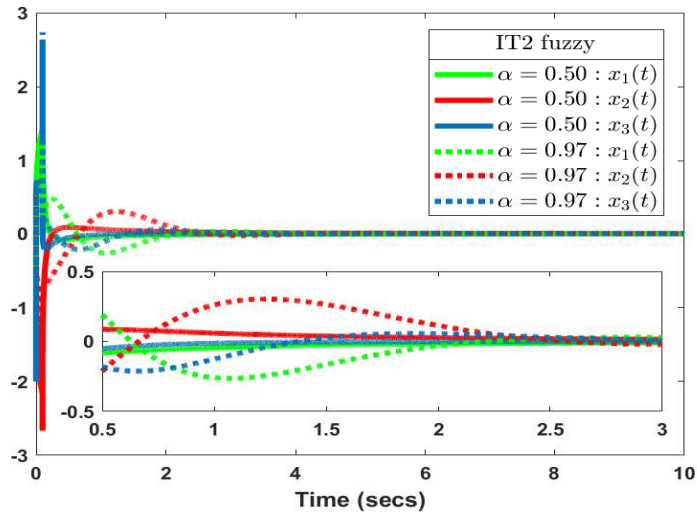

Fig. 13 Error responses for FO $\alpha=0.50$ and $\alpha=0.97$

where $\rho(t)=\left[\rho_{1}(t), \rho_{2}(t), \rho_{3}(t)\right]^{T}$,

$\mathcal{A}_{1}=\left[\begin{array}{ccc}0 & -1 & -1 \\ 1 & s_{1} & 0 \\ s_{2} & 0 & -s_{4}\end{array}\right], \mathcal{A}_{2}=\left[\begin{array}{ccc}0 & -1 & -1 \\ 1 & s_{1} & 0 \\ s_{2} & 0 & s_{4}\end{array}\right]$,

$\mathcal{B}_{1}=\mathcal{B}_{2}=\left[\begin{array}{lll}1 & 0 & 0 \\ 0 & 1 & 0 \\ 0 & 0 & 1\end{array}\right]$, and $\mathcal{C}_{1}=\mathcal{C}_{2}=\left[\begin{array}{l}0 \\ 0 \\ 1\end{array}\right]$.

The aforementioned FO fuzzy system (33) is considered with the type-1 fuzzy MFs in [22] with $s_{1}=0.34, s_{2}=0.4$, $s_{3}=5$ and $s_{4}=10$ and the type-2 fuzzy MFs in [14]. The type-1 fuzzy MFs are chosen as $\lambda_{1}(t)=\frac{1}{2}\left(1+\frac{s_{3}-\rho_{1}(t)}{s_{4}}\right)$ and $\lambda_{2}(t)=1-\lambda_{1}(t)$. Here, we assume that $s_{3}$ is an uncertain parameter and it is denoted as $s_{3}=s_{3}(t) \in[0,5]$. On the other hand, the UBs and LBs of fuzzy MFs for the type2 fuzzy system are given in Table 4 and the MFs for the controller (5) are given in Table 1 . Further, the parameters of fuzzy reference model are taken as follows:

$\mathcal{A}_{1 m}=\left[\begin{array}{ccc}1 & 0 & 0 \\ 0 & 1 & -4 \\ 0 & 1 & -2\end{array}\right], \mathcal{A}_{2 m}=\left[\begin{array}{ccc}-3 & 0 & 5 \\ 0 & -2 & -3 \\ 0 & 0 & -1\end{array}\right]$, and

$\mathcal{B}_{1 m}=\mathcal{B}_{2 m}=\left[\begin{array}{l}0 \\ 0 \\ 1\end{array}\right]$.

Table 4 Plant's membership functions

\begin{tabular}{c|c}
\hline \hline LBs & UBs \\
\hline \hline$\underline{\mu}_{\mathbb{E}_{1}^{1}}\left(\rho_{1}\right)=\frac{1}{2}\left(1+\frac{0-\rho_{1}}{s_{4}}\right)$ & $\bar{\mu}_{\mathbb{E}_{1}^{1}}\left(\rho_{1}\right)=\frac{1}{2}\left(1+\frac{5-\rho_{1}}{s_{4}}\right)$ \\
$\underline{\mu}_{\mathbb{E}_{1}^{2}}\left(\rho_{1}\right)=\frac{1}{2}\left(1-\frac{5-\rho_{1}}{s_{4}}\right)$ & $\bar{\mu}_{\mathbb{E}_{1}^{2}}\left(\rho_{1}\right)=\frac{1}{2}\left(1-\frac{0-\rho_{1}}{s_{4}}\right)$ \\
\hline \hline
\end{tabular}

We choose the external disturbance as $\beta(t)=1.2 \sin (t)$, the reference input as $r(t)=\frac{0.1 \sin (t)}{1+0.01 t}$, and $\mathcal{T}_{k}=0.001$. The choice of the initial condition for the system states and the reference states are taken as $\rho_{1}(0)=1, \rho_{2}(0)=-1$,

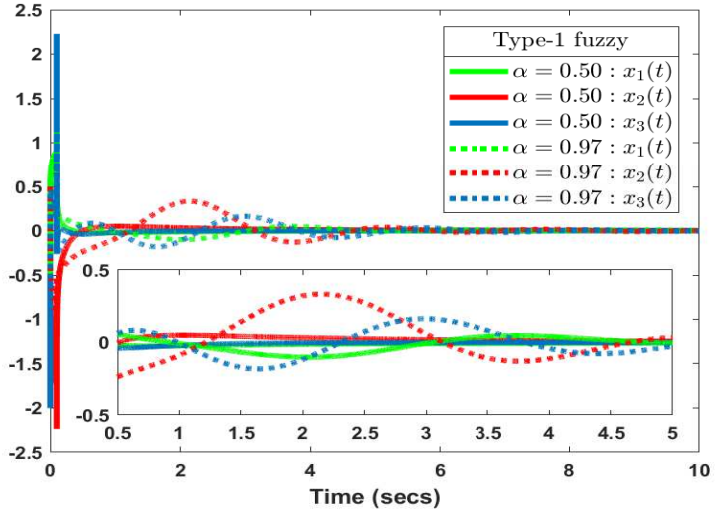

$\rho_{3}(0)=2.5$, and $\rho_{1 m}(0)=0.5, \rho_{2 m}(0)=-0.5, \rho_{3 m}(0)=$ 0.5 , respectively. By solving the conditions obtained in Corollary 1 with the values of the parameters referred to above, a set of feasible matrices are found subsequently from which the associated UDE-based controller gain values are calculated as

$\begin{aligned} \mathcal{K}_{1}= & {\left[\begin{array}{ccc}-3.7069 & 2.8351 & 2.2129 \\ -0.2079 & -1.2172 & 2.4178 \\ -0.1623 & 3.1626 & -1.2291\end{array}\right], } \\ \mathcal{K}_{2}= & {\left[\begin{array}{ccc}-3.6646 & 2.8352 & 2.1596 \\ -0.2209 & -1.1598 & 2.4144 \\ -0.1510 & 3.1798 & -1.2708\end{array}\right] . }\end{aligned}$

Based on the boundary information of the MFs, we obtain the membership function-dependent stabilization conditions for reducing conservativeness. Once we ignored the upper and lower MFs information in Corollary 1, we can obtain the stabilization conditions corresponding to FO type-1 fuzzy system. By solving the obtained LMIs, we can get the following gain matrices:

$\begin{aligned} & \mathcal{K}_{1}= {\left[\begin{array}{ccc}-1.3333 & 0.0000 & 0.0000 \\ 0.0000 & -1.3333 & 1.5000 \\ -0.0000 & 1.5000 & 1.6667\end{array}\right], } \\ & \mathcal{K}_{2}=\left[\begin{array}{ccc}2.6667 & 0.0000 & -2.5000 \\ 0.0000 & 1.6667 & 1.5000 \\ -2.5000 & 1.5000 & 0.6667\end{array}\right] .\end{aligned}$

The tracking error trajectories of the closed-loop system under type-1 and IT2FS approach are displayed in Fig. 13. It is clearly seen that the overshoot of the error trajectory under the IT2FS model is smaller than of the type-1 fuzzy model. It indicates the superiority of the IT2FS design.

It can be seen from above four examples that the proposed UDE-based controller guaranteed the asymptotic tracking performance of the FO IT2FSs (2) and also effectively estimated. 


\section{Conclusion}

This paper have been introduced the UDE-based fuzzy tracking controller for FO IT2FSs. Firstly, the UDE-based control problem has been formulated, in which the proposed control design is split into two control problems, namely the stability of the nominal error dynamic and the unknown lumped uncertainty estimation problem. By employing Lyapunov stability and fractional calculus theories required asymptotic stability criterion for closed-loop error system have been formulated in the form of LMIs. Further, by selecting appropriate filter parameters, the unknown terms in the dynamics of the considered system have been estimated and compensated. Finally, four numerical examples have been provided to demonstrate the effectiveness of the designed robust tracking controller. The workability about UDE-based control law has been demonstrated by the FO IT2FSs. In the future, we shall extend the proposed UDE-based control method to FO fuzzy neural network systems with actuator faults and input saturation [43,44].

\section{Acknowledgements}

The work of first, third and fourth authors was supported by Basic Science Research Program through the National Research Foundation of Korea (NRF) funded by the Ministry of Education (NRF-2020R1A6A1A12047945).

\section{Compliance with ethical standards}

Conflict of interest The authors declare that there is no conflict of interest.

Availability of data and material Data sharing not applicable to this article as no datasets were generated or analysed during the current study.

\section{References}

1. Wu, L.B., Park Ju H., Xie, X.P., Ren, Y.W., Yang.: Distributed adaptive neural network consensus for a class of uncertain nonaffine nonlinear multi-agent systems. Nonlinear Dyn. 100, 12431255(2020)

2. Li, X.J., Yang, G.H.: Robust adaptive fault-tolerant control for a class of uncertain nonlinear time delay systems. IEEE Trans. Syst. Man Cybern. Syst. 47(7), 1554-1563(2017)

3. Wang, H., Shi, P., Li, H., Zhou, Q.: Adaptive neural tracking control for a class of nonlinear systems with dynamic uncertainties. IEEE Trans. Cybern. 47(10), 3075-3087(2017)

4. Xing, L., Wen, C., Liu, Z., Su, H., Cai, J.: Event-triggered adaptive control for a class of uncertain nonlinear systems. IEEE Trans. Autom. control. 62(4), 2071-2076(2017)

5. Chadli, M., Karimi, H.: Robust observer design for unknown inputs Takagi-Sugeno models. IEEE Trans. Fuzzy Syst. 21(1), 158164(2013)
6. Cheema, M.A.M., Fletcher, J.E., Farshadnia, M., Rahman, M.F.: Sliding mode based combined speed and direct thrust force control of linear permanent magnet synchronous motors with first-order plus integral sliding condition. IET Power Electron. 34(3), 25262538(2019)

7. Choi, Y.S., Choi, H.H., Jung, J.W.: Feedback linearization direct torque control with reduced torque and flux ripples for IPMSM drives. IEEE Trans. Power Electron. 31(5), 3728-3737(2016)

8. Takagi T., Sugeno, M.: Fuzzy identification of systems and its applications to modeling and control. IEEE Trans. Syst. Man Cybern. 15(1), 116-132(1985)

9. Li, L., Chadli, M., Ding, S.X., Qiu, J., Yang, Y.: Diagnostic observer design for T-S fuzzy systems: application to real-timeweighted fault-detection approach. IEEE Trans. Fuzzy Syst. 26(2), 805-816(2018)

10. Wang, B., Zhang, D., Cheng J., Park Ju H.: Fuzzy model-based nonfragile control of switched discrete-time systems. Nonlinear Dyn. 93, 2461-2471(2018)

11. Song, J., Niu, Y., Lam, J., Lam, H.K., Fuzzy remote tracking control for randomly varying local nonlinear models under fading and missing measurements. IEEE Trans. Fuzzy Syst. 26(3), 11251237(2018)

12. Wang, Y., Xia, Y., Ahn, C.K., Zhu, Y.: Exponential stabilization of Takagi-Sugeno fuzzy systems with aperiodic sampling: An aperiodic adaptive event-triggered method. IEEE Trans. Syst. Man Cybern. 49(2), 444-454(2019)

13. Lu, Z., Ran, G., Xu, F., Lu J.: Novel mixed-triggered filter design for interval type-2 fuzzy nonlinear Markovian jump systems with randomly occurring packet dropouts. Nonlinear Dyn. 97, 15251540(2019)

14. Li, H., Wang, J., Lam, H.K., Zhou, Q., Du, H.: Adaptive sliding mode control for interval type-2 fuzzy systems. IEEE Trans. Syst. Man Cybern. Syst. 46(12), 1654-1663(2016)

15. Hassani, H., Zarei, J., Chadli, M., Qiu, J.: Unknown input observer design for interval type-2 T-S fuzzy systems with immeasurable premise variables. IEEE Trans. Cybern. 47(9), 2639-2650(2017)

16. Lam, H.K., Li. H., Deters, C., Secco, E.L., Wurdemann, H.A., Althoefer, K.: Control design for interval type-2 fuzzy systems under imperfect premise matching. IEEE Trans. Ind. Electron. 61(2), 956-968(2014)

17. Li, H., Wu, C., Jing, X., Wu, L.: Security control of interval type-2 fuzzy system with two-terminal deception attacks under premise mismatch. Nonlinear Dyn. 102, 431-453(2020)

18. Li, H., Wu, C., Shi, P., Gao, Y.: Control of nonlinear networked systems with packet dropouts: interval type-2 fuzzy model-based approach. IEEE Trans. Cybern. 45(11), 2378-2389(2015)

19. Wu, S.L., Khaleel, M.A.: Parameter optimization in waveform relaxation for fractional-order $R C$ circuits. IEEE Trans. Circuits Syst. I Reg. Papers. 64(7), 1781-1790(2017)

20. Xiao, M., Zheng, W.X., Jiang, G., Cao, J.: Undamped oscillations generated by Hopf bifurcations in fractional-order recurrent neural networks with Caputo derivative. IEEE Trans. Neural Netw. learn. Syst. 26(12), 3201-3214(2015)

21. Bao, H., Park, JU H., Cao, J.: Synchronization of fractional-order complex-valued neural networks with time delay. Neural Netw. 81, 16-28(2016)

22. Li, Y., Li, J.: Stability analysis of fractional order systems based on T-S fuzzy model with the fractional order $\alpha: 0<\alpha<1$. Nonlinear Dyn. 78(4), 2909-2919(2014)

23. Podlubny, I.: Fractional Differential Equations. Academic Press. New York.(1999)

24. Sun, G., Ma, Z.: Practical tracking control of linear motor with adaptive fractional order terminal sliding mode control. IEEE/ASME Trans. Mechatronics. 22(6), 2643-2653(2017)

25. Tan, Y., Xiong, M., Du, D., Fei, S.: Observer-based robust control for fractional-order nonlinear uncertain systems with input saturation and measurement quantization. Nonlinear Anal. Hybrid Syst. 34, 45-57(2019) 
26. Wang, J., Ye, Y., Pan, X., Gao, X., Zhuang, C.: Fractional zerophase filtering based on the Riemann-Liouville integral. Signal Processing. 98, 150-157(2014)

27. Jafari, P., Teshnehlab, M., Kakhki, M.T.: Adaptive type-2 fuzzy system for synchronisation and stabilisation of chaotic non-linear fractional order systems. IET Control Theory Appl. 12(2), 183193(2018)

28. Wang, B., Liu, Z., Li, S.E., Moura, S.J., Peng, H.: State-of-charge estimation for lithium-ion batteries based on a nonlinear fractional model. IEEE Trans. Control Syst. Technol. 25(1), 3-11(2017)

29. Khalil, H.K., Praly, L.: High-gain observers in nonlinear feedback control. Int. J. Robust Nonlinear Control. 24(6), 993-1015(2014)

30. Li, H., Sun, Z., Liu, H., Sun, F., Deng, J.: State feedback integral control of networked control systems with external disturbance. IET Control Theory Appl. 5(2), 283-290(2011)

31. Park, M.J., Kwon, O.M., Park, Ju H., Lee, S.M., Cha, E.J.: $H_{\infty}$ state estimation for discrete-time neural networks with interval time-varying delays and probabilistic diverging disturbances. Neurocomputing. 153, 255-270(2015)

32. Wang, L., Wang, Z., Han, Q.L., Wei, G.: Synchronization control for a class of discrete-time dynamical networks with packet dropouts: A coding-decoding-based approach. IEEE Trans. Cybern. 48(8), 2437-2448(2018)

33. J. Yang, J. Su, S. Li, and X. Yu, High-order mismatched disturbance compensation for motion control systems via a continuous dynamic sliding-mode approach. IEEE Trans. Ind. Inf. 10(1), 604614(2014)

34. Li, S., Yang, J., Chen, WH., Chen, X.: Disturbance observerbased control: methods and applications. CRC Press. Boca Rato.(2017)

35. Ren, B., Zhong, Q.C., Chen, J.: Asymptotic reference tracking and disturbance rejection of UDE-based robust control. IEEE Trans. Ind. Electron. 64(4), 3166-3176(2017)

36. Zhong, Q.C., Rees, D.: Control of uncertain LTI systems based on an uncertainty and disturbance estimator. ASME J. Dyn. Sys. Meas. Control. 126(4), 905-910(2004)

37. Chen, J., Ren, B., Zhong, Q.C.: UDE-based trajectory tracking control of piezoelectric stages. IEEE Trans. Ind. Electron. 63(10), 6450-6459(2016)

38. Chen, L., Wu, R., Cheng, Y., Chen, Y., Zhuang, C.: Delaydependent and order-dependent stability and stabilization of fractional-order linear systems with time-varying delay. IEEE Trans. Circuits Syst. II Reg. Papers. 67, 1064-1068(2020)

39. Boyd, S, Ghaoui, L.EL., Feron, E., Balakrishnan, V.: Linear Matrix Inequalities in System and Control Theory. SIAM. Philadelphia.(1994)

40. Zhang. X., Huang, W., Wang, Q.G.: Robust $H_{\infty}$ adaptive sliding mode fault tolerant control for T-S fuzzy fractional order systems with mismatched disturbances, IEEE Trans. Circuits Syst. I Reg. Papers. 68(3), 1297-1307(2021)

41. Shanmugam, S., Joo, Y.H.: Design of interval type-2 fuzzy-based sampled-data controller for nonlinear systems using novel fuzzy Lyapunov functional and its application to PMSM, IEEE Trans. Syst. Man Cybern. Syst.. 51(1), 1297-1307(2021)

42. Toumi, Y.K., Ito, O.: A time delay controller for systems with unknown dynamics, ASME J. Dyn. Syst. Meas. Control. 112(1), 133142(1990)

43. Song, S., Zhang. B., Song, X., Zhang, Z.: Neuro-fuzzy-based adaptive dynamic surface control for fractional-order nonlinear strict-feedback systems with input constraint. IEEE Trans. Syst. Man Cybern. Syst. (2019) DOI: 10.1109/TSMC.2019.2933359

44. Song, S., Park. J.H., Zhang, B., Song, X.: Observer-based adaptive hybrid fuzzy resilient control for fractional-order nonlinear systems with time-varying delays and actuator failures. IEEE Trans. Fuzzy Syst. 29(3), 471-485(2021) 